\title{
Evaluation of Dynamic Characteristics for a Submerged Body with Large Angle of Attack Motion via CFD Analysis
}

\author{
Myungjun Jeon ${ }^{\oplus 1}$, Thi Loan Mai®1, Hyeon Kyu Yoon ${ }^{\oplus^{2}}$, Jaekwan Ryu ${ }^{\oplus^{3}}$, Wonhee Lee ${ }^{\oplus^{3}}$ and Pyungmo $\mathrm{Ku}^{{ }^{4}}$ \\ ${ }^{1}$ Ph.D. Candidante Student, Department, of Eco-friendly Offshore Plant FEED Engineering, Changwon National University, Korea \\ ${ }^{2}$ Professor, Department, of Naval Architecture \& Marine Engineering, Changwon National University, Korea \\ ${ }^{3}$ Chief Researcher, LIG Nexl Unmanned Systems R\&D Laboratory, Korea \\ ${ }^{4}$ Senior Researcher, LIG Nexl Unmanned Systems R\&D Laboratory, Korea
}

KEY WORDS: Submerged body, Computational fluid dynamics, Large angle of attack, Virtual captive model tests, Maneuvering simulation

ABSTRACT: A submerged body with varied control inputs can execute large drift angles and large angles of attack, as well as basic control such as straight movement and turning. The objective of this study is to analyze the dynamic characteristics of a submerged body comprising six thrusters and six control planes, which is capable of a large drift angle and angle of attack motion. Virtual captive model tests via were analyzed via computational fluid dynamics (CFD) to determine the dynamic characteristics of the submerged body. A test matrix of virtual captive model tests specialized for large-angle motion was established. Based on this test matrix, virtual captive model tests were performed with a drift angle and angle of attack of approximately $30^{\circ}$ and $90^{\circ}$, respectively. The characteristics of the hydrodynamic force acting on the horizontal and vertical surfaces of the submerged body were analyzed under the large-angle motion condition, and a model representing this hydrodynamic force was established. In addition, maneuvering simulation was performed to evaluate the standard maneuverability and dynamic characteristics of large-angle motion. Considering the shape characteristics of the submerged body, we attempt to verify the feasibility of the analysis results by analyzing the characteristics of the hydrodynamic force when the large-angle motion occurred.

\begin{tabular}{|c|c|c|c|}
\hline \multicolumn{2}{|r|}{ Nomenclature } & $X_{u}$ & Derivative of $X_{H D}$ with respect to $u$ \\
\hline & & $X_{u|u|}$ & Derivative of $X_{H D}$ with respect to $u|u|$ \\
\hline$O-x y z$ & Earth-fixed coordinate & $X_{\delta_{b}\left|\delta_{b}\right|}$ & Derivative of $X_{C}$ with respect to $\delta_{b}\left|\delta_{b}\right|$ \\
\hline$o-x_{b} y_{b} z_{b}$ & Body-fixed coordinate & $X_{\delta_{s}\left|\delta_{s}\right|}$ & Derivative of $X_{C}$ with respect to $\delta_{s}\left|\delta_{s}\right|$ \\
\hline$U$ & Vehicle speed $(\mathrm{m} / \mathrm{s})$ & $X_{\delta_{r}\left|\delta_{r}\right|}$ & Derivative of $X_{C}$ with respect to $\delta_{r}\left|\delta_{r}\right|$ \\
\hline$\beta$ & Drift angle (deg) & & \\
\hline$\alpha$ & Angle of attack (deg) & $Y_{\dot{v}}$ & Derivative of $Y_{H D}$ with respect to $v$ \\
\hline$u$ & Surge (axial) velocity $(\mathrm{m} / \mathrm{s})$ & $Y_{\dot{p}}$ & Derivative of $Y_{H D}$ with respect to $\dot{p}$ \\
\hline$v$ & Sway (lateral) velocity (m/s) & $Y_{\dot{r}}$ & Derivative of $Y_{H D}$ with respect to $\dot{r}$ \\
\hline$w$ & Heave (vertical) velocity $(\mathrm{m} / \mathrm{s})$ & $Y_{v}$ & Derivative of $Y_{H D}$ with respect to $v$ \\
\hline$p$ & Roll rate (deg/s) & $Y_{p}$ & Derivative of $Y_{H D}$ with respect to $p$ \\
\hline$q$ & Pitch rate $(\mathrm{deg} / \mathrm{s})$ & $Y_{r}$ & Derivative of $Y_{H D}$ with respect to $r$ \\
\hline$r$ & Yaw rate $(\mathrm{deg} / \mathrm{s})$ & $Y_{\delta_{r}}$ & Derivative of $Y_{C}$ with respect to $\delta_{r}$ \\
\hline$\delta_{r}$ & Rudder angle (deg) & $Z_{\dot{w}}$ & Derivative of $Z_{H D}$ with respect to $\dot{w}$ \\
\hline$\delta_{b}$ & Bow plane angle (deg) & $Z_{\dot{q}}$ & Derivative of $Z_{H D}$ with respect to $\dot{q}$ \\
\hline$\delta_{s}$ & Stern plane angle (deg) & $Z_{w}$ & Derivative of $Z_{H D}$ with respect to $w$ \\
\hline$X_{\dot{u}}$ & Derivative of $X_{H D}$ with respect to $\dot{u}$ & $Z_{q}$ & Derivative of $Z_{H D}$ with respect to $q$ \\
\hline
\end{tabular}

Received 20 July 2021, revised 31 July 2021, accepted 2 August 2021

Corresponding author Hyeon Kyu Yoon: +82-55-213-3683, hkyoon@changwon.ac.kr

(C) 2021, The Korean Society of Ocean Engineers

This is an open access article distributed under the terms of the creative commons attribution non-commercial license (http://creativecommons.org/licenses/by-nc/4.0) which permits unrestricted non-commercial use, distribution, and reproduction in any medium, provided the original work is properly cited 


$\begin{array}{ll}Z_{\delta_{b}} & \text { Derivative of } Z_{C} \text { with respect to } \delta_{b} \\ Z_{\delta_{s}} & \text { Derivative of } Z_{C} \text { with respect to } \delta_{s} \\ K_{\dot{v}} & \text { Derivative of } K_{H D} \text { with respect to } \dot{v} \\ K_{\dot{p}} & \text { Derivative of } K_{H D} \text { with respect to } \dot{p} \\ K_{\dot{r}} & \text { Derivative of } K_{H D} \text { with respect to } \dot{r} \\ K_{v} & \text { Derivative of } K_{H D} \text { with respect to } v \\ K_{p} & \text { Derivative of } K_{H D} \text { with respect to } p \\ K_{r} & \text { Derivative of } K_{H D} \text { with respect to } r \\ K_{\delta_{r}} & \text { Derivative of } K_{C} \text { with respect to } \delta_{r} \\ M_{\dot{w}} & \text { Derivative of } M_{H D} \text { with respect to } \dot{w} \\ M_{q} & \text { Derivative of } M_{H D} \text { with respect to } \dot{q} \\ M_{w} & \text { Derivative of } M_{H D} \text { with respect to } w \\ M_{q} & \text { Derivative of } M_{H D} \text { with respect to } q \\ M_{\delta_{b}} & \text { Derivative of } M_{C} \text { with respect to } \delta_{b} \\ M_{\delta_{s}} & \text { Derivative of } M_{C} \text { with respect to } \delta_{s} \\ N_{\dot{v}} & \text { Derivative of } N_{H D} \text { with respect to } \dot{v} \\ N_{\dot{p}} & \text { Derivative of } N_{H D} \text { with respect to } \dot{p} \\ N_{\dot{r}} & \text { Derivative of } N_{H D} \text { with respect to } \dot{r} \\ N_{v} & \text { Derivative of } N_{H D} \text { with respect to } v \\ N_{p} & \text { Derivative of } N_{H D} \text { with respect to } p \\ N_{r} & \text { Derivative of } N_{H D} \text { with respect to } r \\ N_{\delta_{r}} & \text { Derivative of } N_{C} \text { with respect to } \delta_{r}\end{array}$

\section{Introduction}

A submerged body refers to all vehicles operated in water, such as a remotely operated vehicles (ROVs), autonomous underwater vehicles (AUVs), underwater weapons, and submarines (Kim et al., 2012; Park et al., 2015). Different types of submerged bodies serve varying purposes in military or commercial applications. Because submerged bodies have distinct operational purposes, their dynamic characteristics must reflect their operational purpose, and their shapes must be designed to satisfy the required dynamic characteristics accordingly. Therefore, a submerged body must be developed with a suitable performance for each task and utilization (Jeon et al., 2017). In particular, it is difficult to monitor the state of a small submerged body with the human eye because it is often remotely controlled or autonomously driven, unlike vessels manned by humans. Therefore, the maneuverability of such a submerged body needs to be sufficiently examined during the design stage to ensure the adoption of an appropriate controller.

Studies based on experimental and analytical methods have been actively conducted on estimating the maneuverability of a submerged body. The most accurate maneuverability analysis in the design stage involves modeling the external force of maneuvering equations of motion that reflect the motion range and operational purpose of a submerged body and estimating the external force model coefficients by performing a captive model test in a towing tank. Bae and Sohn (2009) conducted a static test including a resistance test in a circular water channel for Manta-type unmanned underwater vehicles. The maneuvering equation of motion with six degrees of freedom for Manta-type unmanned underwater vehicles was established by combining certain damping coefficients determined via a static test with added mass and damping coefficients estimated theoretically, and then, maneuverability was analyzed. Kim et al. (2012) conducted a captive model test by installing a submerged body model in a large-scale controlled computerized planar motion carriage. In addition to stability analysis, resistance, static drift, static angle of attack, horizontal and vertical turning, and combined tests were performed to estimate a damping coefficient, excluding the added mass coefficient. Jung et al. (2014) estimated added mass and damping coefficients by performing a vertical planar motion mechanism test in a linear towing tank for an autonomous underwater glider. In addition, they modeled the equation of motion based on the estimated coefficients to be adopted as the plant model for a control simulation. Owing to the cylindrical shape of most submerged bodies, the roll hydrodynamic damping moment is relatively small, and it triggers a large rolling motion at a high speed if the rolling motion is not properly controlled. To estimate a rolling motion of a sensitive submerged body, Park et al. (2015) conducted a coning motion test to estimate the hydrodynamic derivative, which is related to rolling motion, and proposed a different coning motion test method. The aforementioned model test cases were conducted for submerged bodies with slender shapes, under the assumption that the drift angle and angle of attack were not large at the design speed; hence, additional research is required to reflect a large angle of attack motion.

Estimating the maneuverability of a submerged body via analytical methods can be divided into two categories. The simplest approach adopts an empirical formula. Yeo et al. (2006) deduced the effect of design factors on the stability of a submarine based on an empirical formula that assumes that the main body is a lift force generator, which they substituted with an equivalent wing. Jeon et al. (2017) analyzed the effects of shape design factors on the maneuverability of a submerged body. However, an empirical formula was used to establish a plant model for designing a controller and estimating a linear stability coefficient for stability analysis. Simulating the large attack angle motion and slow motion is limited because a nonlinear coefficient cannot be estimated. The other approach involves conducting virtual captive model tests based on computational fluid dynamics (CFD). Recently, estimating hydrodynamic derivatives by performing a CFD in the same condition as a captive model test conducted in a tank has been widely adopted, and the accuracy results are similar to that of a model test. Sung and Park (2015) conducted a CFD-based virtual captive model test for open merchant vessels, KCS (KRISO Container Ship) and KVLCC (KRISO Very Large Crude-oil Carrier) $1 \& 2$, and compared the accuracy with the free running model test results. In terms of accuracy, They produced outstanding results. Nguyen et al. (2018) calculated the hydrodynamic force acting on a 
full scale submarine using a CFD analysis based on the Reynoldsaveraged Navier-Stokes equations (RANS), and estimated damping coefficients. Cho et al. (2020) performed a virtual captive model test using CFD for submarines equipped with an X-rudder and deduced results similar to an experimental outcome.

In general, a large attack angle motion needs to be considered in a case when a vessel is operated at a low speed when berthing at a port (Takashina, 1986; Yoon and Kim, 2005), or when lateral and vertical movements are free, such as in ROVs (Jeon et al., 2016). In this study, we analyze the dynamic characteristics of a submerged body equipped with six thrusters and six control panels. A submerged body with various control inputs has relatively more free motions than a slender-type submerged body comprising a rudder attached in the rear part, elevator, and thruster. Specifically, motions in large drift angles and large angles of attack are possible. Because the motions are in large drift angles and large angles of attack, the motion range needs to be set broadly to accurately estimate the hydrodynamic force in the analysis.

Maneuverability can be directly investigated if a virtual freerunning model test is conducted using CFD; however, it requires a significant amount of computation resources, and the maneuvering equation of motion, which is a plant model used for designing controllers, cannot be determined. Therefore, a virtual captive model test was performed in this study via CFD analysis to analyze the dynamic characteristics of a submerged body with a large angle of attack motion. This method is more advantageous than a model test in terms of time and cost, and it is also more advantageous than a water vessel in terms of computation time because computation is performed in water instead of on a free surface.

Most previous studies first established a maneuvering mathematical model and then estimated maneuvering coefficients comprising the mathematic model in their analyses. However, it is challenging to establish a mathematical model when the force tendencies are not thoroughly examined, the correlation between forces and motions is difficult to predict, and the data available on the submerged body are insufficient. Therefore, this study first examined the tendency of forces by establishing the conditions of a virtual captive model test in which hydrodynamic forces including large angles of attack motion were estimated and then modeled the correlation between forces and motions.

\section{Coordinate System and Test Conditions}

\subsection{Coordinate System}

Fig. 1 presents a coordinate system comprising the Earth-fixed coordinate for expressing the motion trajectory, orientation angle, and a body-fixed coordinate for defining the equation of motion and external forces acting on a submerged body. All the symbols used in the equation of motion and coordinate system are in accordance with the symbolic notation defined by Fossen (2011).

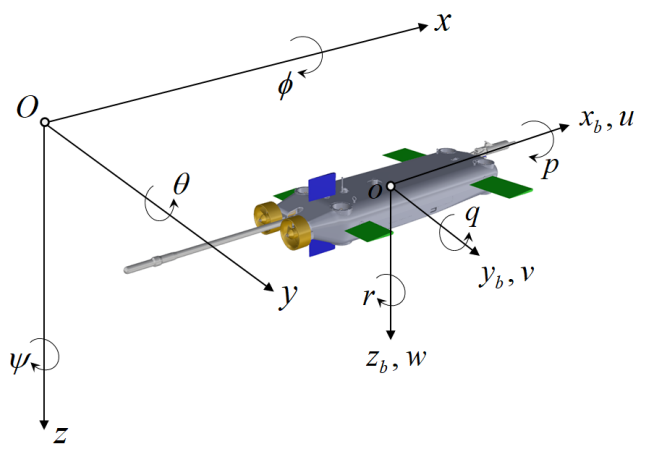

Fig. 1 Coordinate systems

\subsection{Subject Submerged Body}

Fig. 2 shows that the subject submerged body is equipped with two main thrusters in the rear part and four auxiliary thrusters for adjusting the depth, including a rudder for controlling directions, a bow plane for controlling depth, and a rear elevator. Here, six control inputs generate thrust and rudder force based on the large angle of attack and angle of attack motions that are possible. In addition, the principal parameters are presented in Table 1 .

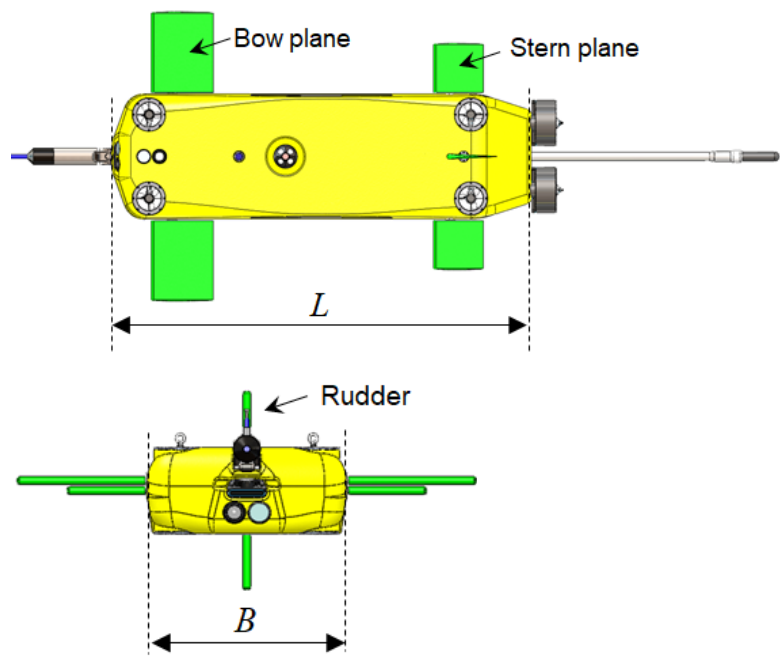

Fig. 2 Shape of a subject submerged body

Table 1 Principal parameters of the subject submerged body

\begin{tabular}{|c|c|c|}
\hline Item (unit) & Symbol & Value \\
\hline Length (m) & $L$ & 2.0 \\
\hline Beam (m) & $B$ & 0.6 \\
\hline Depth (m) & $D$ & 0.26 \\
\hline Volume $\left(\mathrm{m}^{3}\right)$ & $\nabla$ & 0.281 \\
\hline 2-rudder area $\left(\mathrm{m}^{2}\right)$ & $A_{r d}$ & 0.03984 \\
\hline 2-bow plane area $\left(\mathrm{m}^{2}\right)$ & $A_{b p}$ & 0.1158 \\
\hline 2-stern plan area $\left(\mathrm{m}^{2}\right)$ & $A_{s p}$ & 0.0552 \\
\hline $\begin{array}{c}x-, y-, \text { and } z \text {-axis } \\
\text { Center of gravity (m) }\end{array}$ & $\left(\begin{array}{lll}x_{g} & y_{g} & z_{g}\end{array}\right)$ & $(-0.082,0.0,0.0015)$ \\
\hline $\begin{array}{l}x-, y \text {-, and } z \text {-axis } \\
\text { moment of inertia }\left(\mathrm{kg} \cdot \mathrm{m}^{2}\right)\end{array}$ & $\left(\begin{array}{lll}I_{x x} & I_{y y} & I_{z z}\end{array}\right)$ & $(6.08,65.49,69.82)$ \\
\hline
\end{tabular}




\subsection{Test Conditions}

The conditions of the virtual captive model tests were configured, as presented in Table 2. Provided the CFD analysis is performed under the conditions in Table 2, all maneuvering coefficients can be obtained, excluding the coefficient related to thrust. All test conditions except a resistance test are applied with a design speed of $2.57 \mathrm{~m} / \mathrm{s}$ (5 knots). Because there are two main thrusters attached in the rear part, yawing moment can be generated via steering and by thrust. Therefore, the submerged body may have a higher circular angular velocity than a

Table 2 Calculation matrix of virtual captive model tests

\begin{tabular}{|c|c|}
\hline Test type & Motion variables \\
\hline Resistance test & $\begin{array}{l}U=1.03,1.54,2.06,2.57,3.09,3.60 \\
\mathrm{~m} / \mathrm{s}(2.0,3.0,4.0,5.0,6.0,7.0 \mathrm{knot})\end{array}$ \\
\hline Static drift test & $\begin{aligned} \beta= & \pm 2^{\circ}, \pm 4^{\circ}, \pm 6^{\circ}, \pm 8^{\circ}, \pm 10^{\circ}, \pm 12^{\circ} \\
& \pm 15^{\circ}, \pm 20^{\circ}, \pm 25^{\circ}, \pm 30^{\circ}\end{aligned}$ \\
\hline Static angle of attack test & $\begin{aligned} \alpha= & \pm 2^{\circ}, \pm 4^{\circ}, \pm 6^{\circ}, \pm 8^{\circ}, \pm 10^{\circ}, \pm 12^{\circ} \\
& \pm 15^{\circ}, \pm 20^{\circ}, \pm 25^{\circ}, \pm 30^{\circ}, \pm 40^{\circ} \\
& \pm 50^{\circ}, \pm 60^{\circ}, \pm 70^{\circ}, \pm 80^{\circ}, \pm 90^{\circ}\end{aligned}$ \\
\hline $\begin{array}{l}\text { Horizontal circular } \\
\text { motion test }\end{array}$ & $r^{\prime}= \pm 0.2, \pm 0.3, \pm 0.4, \pm 0.5, \pm 0.6$ \\
\hline $\begin{array}{l}\text { Vertical circular motion } \\
\text { test }\end{array}$ & $q^{\prime}= \pm 0.2, \pm 0.3, \pm 0.4, \pm 0.5, \pm 0.6$ \\
\hline $\begin{array}{l}\text { Horizontal circular } \\
\text { motion with drift test }\end{array}$ & $\begin{array}{c}\beta= \pm 2^{\circ}, \pm 4^{\circ}, \pm 6^{\circ},+8^{\circ},+10^{\circ},+12^{\circ} \\
r^{\prime}=0.2,0.3,0.4,0.5\end{array}$ \\
\hline $\begin{array}{l}\text { Vertical circular motion } \\
\text { with angle of attack test }\end{array}$ & $\begin{array}{c}\alpha= \pm 2^{\circ}, \pm 4^{\circ}, \pm 6^{\circ},-8^{\circ},-10^{\circ},-12^{\circ} \\
q^{\prime}=0.2,0.3,0.4,0.5\end{array}$ \\
\hline Static rudder test & $\begin{aligned} \delta_{r}= \pm 3^{\circ}, & \pm 6^{\circ}, \pm 9^{\circ}, \pm 12^{\circ}, \pm 15^{\circ} \\
& \pm 18^{\circ}, \pm 21^{\circ}\end{aligned}$ \\
\hline $\begin{array}{l}\text { Static elevator test } \\
\text { (bow plane) }\end{array}$ & $\begin{aligned} \delta_{b}= \pm 3^{\circ}, & \pm 6^{\circ}, \pm 9^{\circ}, \pm 12^{\circ}, \pm 15^{\circ} \\
& \pm 18^{\circ}, \pm 21^{\circ}\end{aligned}$ \\
\hline $\begin{array}{l}\text { Static elevator test } \\
\text { (stern plane) }\end{array}$ & $\begin{aligned} \delta_{s}= \pm 3^{\circ}, & \pm 6^{\circ}, \pm 9^{\circ}, \pm 12^{\circ}, \pm 15^{\circ} \\
& \pm 18^{\circ}, \pm 21^{\circ}\end{aligned}$ \\
\hline Roll rotating test & $p^{\prime}=0.4,0.5,0.6,0.7$ \\
\hline Pure sway test & $\dot{v}^{\prime}=0.08,0.16,0.24,0.32$ \\
\hline Pure yaw test & $\dot{r}^{\prime}=0.29,0.52,0.76,1.02$ \\
\hline Pure heave test & $\dot{w}^{\prime}=0.08,0.16,0.24,0.32$ \\
\hline Pure pitch test & $\dot{q}^{\prime}=0.29,0.52,0.76,1.02$ \\
\hline Pure roll test & $\dot{p}^{\prime}=7.2,14.3,21.5,28.6,35.8$ \\
\hline
\end{tabular}

Table 3 Analytic methods and conditions for CFD calculation

\begin{tabular}{cc}
\hline Item & Description \\
\hline Turbulence model & $k-\omega$ model \\
Algorithm & $\begin{array}{c}\text { Semi-implicit method for } \\
\text { pressure-linked equations (SIMPLE) }\end{array}$ \\
$\begin{array}{c}\text { Interpolation method for } \\
\text { pressure }\end{array}$ & Second order upwind \\
Number of elements & $7,070,460$ \\
Number of nodes & $1,309,828$ \\
Type of mesh & $\begin{array}{c}\text { Tetrahedral unstructured mesh, } \\
\text { Prism layer mesh (near hull surface) }\end{array}$ \\
\hline
\end{tabular}

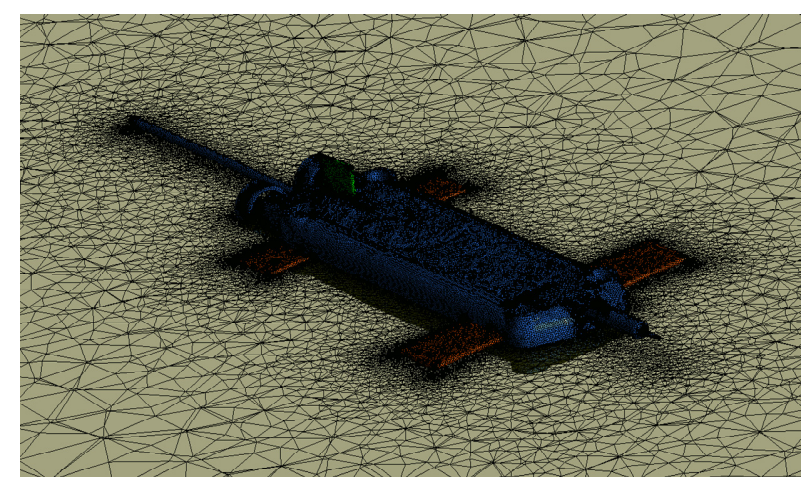

Fig. 3 Mesh generation

submerged body that simply turns by steering, and the drift angle may also be larger because the circular angular velocity and drift angle are linked. Accordingly, a drift angle of approximately $\pm 30^{\circ}$ is considered when conducting a static drift test, as well as a dimensionless angular velocity of approximately 0.6. For self-propulsion, four auxiliary thrusters are attached to the hull, in addition to the main thrusters, and thrust can be freely generated in the vertical direction. Computation conditions were established considering an angle of attack of approximately $\pm 90^{\circ}$ because heave motions were realized by auxiliary thrusters. Hydrodynamic forces were computed using ANSYS FLUENT version 20.1, which is a commercial CFD analysis program. The analysis conditions adopted are presented in Table 3, and Fig. 3 illustrates the results obtained from mesh generation.

The $k-\omega$ model is widely used to predict hydrodynamic forces applied on maneuvering ships (Quérard et al., 2008). The $k-\omega$ model for hydrodynamic derivatives is advantageous in terms of its CPU computation time. Because CFD calculations under several conditions are required in this study, the $k-\omega$ model was selected as the turbulence model. A 3D incompressible viscous flow was assumed, and a continuity equation and RANS equations were applied as governing equations (Jeon et al., 2016). The convection term was applied with the second-order upwind method, diffusion term was discretized using a second-order central differential method, and pressure and speed were linked by the semi-implicit method for pressure-linked equations (SIMPLE).

\section{Analysis Results and Modeling}

\subsection{Static Test}

A static test estimates damping coefficients by calculating the damping force contributing to the speed of the hull and the rotation of a control plane, and it comprises resistance, static drift, static angle of attack, combined, static rudder, and static elevator tests. Details on the hydrodynamic force model of each test are presented in Table 4 . The hydrodynamic force model was divided into two: a linear model in which the linearization of the force is possible because the size of perturbed state variables is small and a nonlinear model considering the nonlinearity of the force generated as motions become greater.

The results of the resistance test for estimating resistance per speed 
are presented in Fig. 4. When neutral buoyancy is assumed as the force proportional to the square of the advanced speed, approximately $9.8 \%$ of dead weight is assumed to be applied in the design speed. Here, dead weight refers to the product of the acceleration of gravity and the mass of the submerged body. Resistance is proportional to the square of the advanced speed, and the resistance applied on the hull can be linearized if the change in the speed is negligible, based on a design speed of $2.57 \mathrm{~m} / \mathrm{s}$ ( 5 knots). Accordingly, Fig. 4 presents the results of the curve approximation of both linear and nonlinear models.

A static drift test calculates the damping force applied when only the sway velocity is generated; the results obtained from calculating the force by adjusting the drift angle by $\pm 30^{\circ}$ are presented in Fig. 5 . Nondimensionalization complies with the prime system 1 defined by the Society of Naval Architecture and Marine Engineering (SNAME) (Fossen, 2011). The hydrodynamic forces were modeled according to the tendency of forces. The nonlinear model presented in Table 3 was considered appropriate, and the coefficient of determination $R^{2}$ was

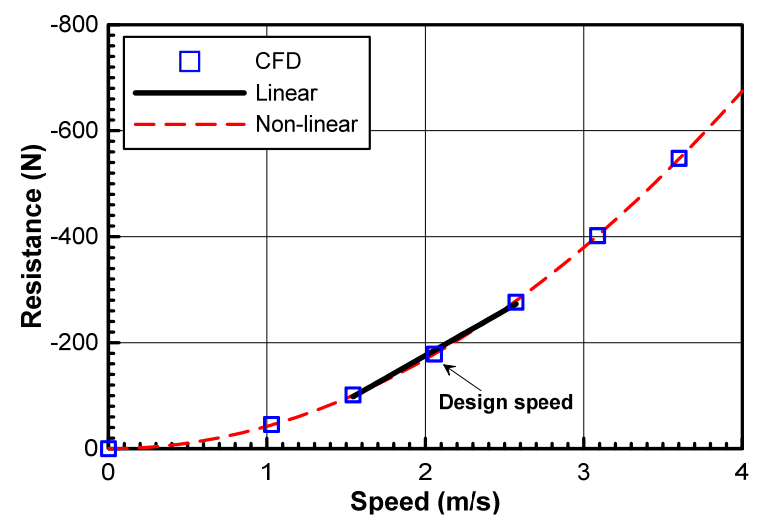

Fig. 4 Result of resistance tests

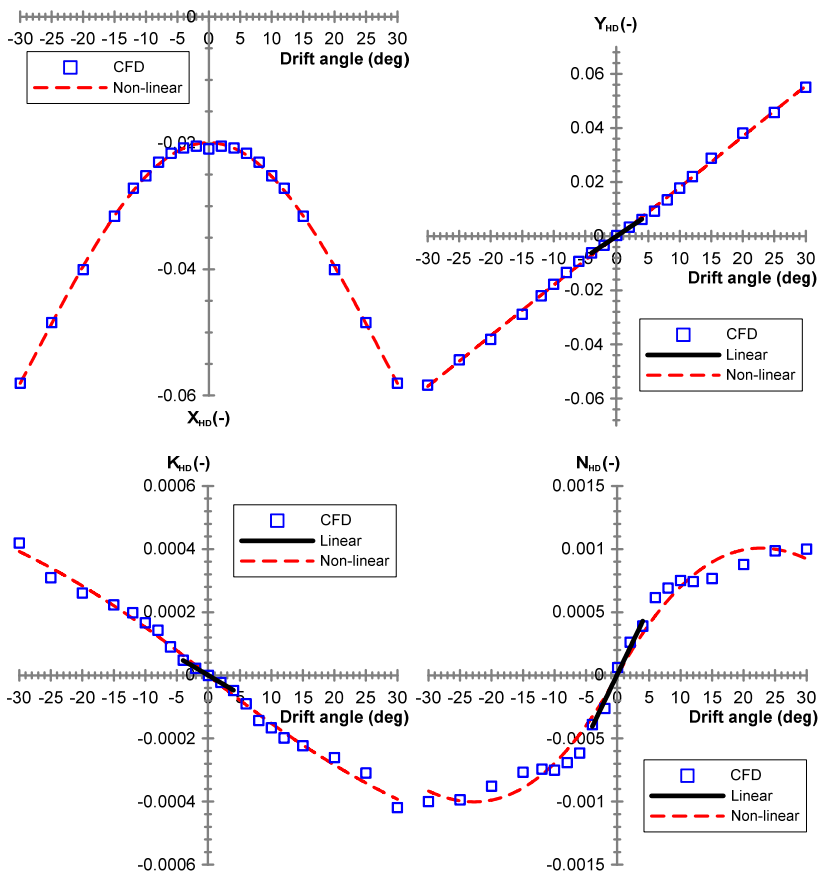

Fig. 5 Results obtained from static drift tests
Table 4 Static hydrodynamic force model for hull motion variables

\begin{tabular}{|c|c|c|c|}
\hline Test type & Model & Formulae & $R^{2}$ \\
\hline \multirow{3}{*}{ Resistance } & Linear & $X_{H D}=X_{0}+X_{u} u$ & $R_{X}^{2}=0.9868$ \\
\hline & $\begin{array}{l}\text { Non- } \\
\text { linear }\end{array}$ & $X_{H D}=X_{0}+X_{u|u|} u|u|$ & $R_{X}^{2}=0.9999$ \\
\hline & Linear & $\left\{\begin{array}{l}X_{H D}=X_{0} \\
Y_{H D}=Y_{0}+Y_{v} v \\
K_{H D}=K_{0}+K_{v} v,-4^{\circ}<\beta<4^{\circ} \\
N_{H D}=N_{0}+N_{v} v\end{array}\right.$ & $\begin{array}{l}R_{Y}^{2}=0.9983 \\
R_{K}^{2}=0.9904 \\
R_{N}^{2}=0.9771\end{array}$ \\
\hline
\end{tabular}

Static drift

Non-

$\left(X_{H D}=X_{0}+X_{v v} v^{2}+X_{v v v v} v^{4} \quad R_{X}^{2}=0.9969\right.$

linear

$\left\{\begin{array}{l}X_{H D}=X_{0}+X_{v v}=Y_{0}+Y_{v} v+Y_{v|v|} v v v \mid \\ Y_{H D}=K_{0}+K_{v} v+K_{v|v|} v \mid v \\ N_{H D}=N_{0}+N_{v} v+N_{v v v} v \mid v\end{array}\right.$

$R_{Y}^{2}=0.9999$

$R_{K}^{2}=0.9929$

$R_{N}^{2}=0.9846$

Static

Linear

$\left\{X_{H D}=X_{0}\right.$

angle

of attack

Non-

linear

$\left\{\begin{array}{l}X_{H D}=X_{0} \\ Z_{H D}=Z_{0}+Z_{w} w,-4^{\circ}<\alpha<4^{\circ} \\ M_{H D}=M_{0}+M_{w} w\end{array}\right.$

$R_{Z}^{2}=0.9863$

$R_{M}^{2}=0.9968$

$X_{H D}=X_{0}+X_{w w} w^{2}+X_{w w w w} w^{4}$

$R_{X}^{2}=0.9193$

$R_{Z}^{2}=0.9988$

$\left\{\begin{array}{l}Z_{H D}=Z_{0}+Z_{w} w+Z_{w|w|} w|w| \\ M_{H D}=M_{0}+M_{w} w+M_{w|w|} w|w|\end{array}\right.$

$R_{M}^{2}=0.9758$

Linear

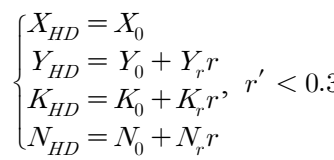

$R_{Y}^{2}=0.9988$

$R_{K}^{2}=0.9987$

$R_{N}^{2}=0.9998$

Horizontal

circular

motion

Non-

$\left\{\begin{array}{l}X_{H D}=X_{0}+X_{r r} r^{2} \\ Y_{H D}=Y_{0}+Y_{r} r+Y_{r|r|} r|r| \\ K_{H D}=K_{0}+K_{r} r+K_{r|r|} r|r| \\ N_{H D}=N_{0}+N_{r} r+N_{r|r|} r|r|\end{array}\right.$

$R_{X}^{2}=0.9810$

$R_{Y}^{2}=0.9966$

$R_{K}^{2}=0.8696$

$R_{N}^{2}=0.9900$

Vertical

Linear

$$
\left\{\begin{array}{l}
X_{H D}=X_{0} \\
Z=Z+Z \\
Z H D
\end{array}\right.
$$

circular

motion

Non-

$\left\{\begin{array}{l}Z_{H D}=Z_{0}+Z_{q} q \\ M_{H D}=M_{0}+M_{q} q\end{array}\right.$

$R_{Z}^{2}=0.9862$

$R_{M}^{2}=0.9998$

$R_{X}^{2}=0.7684$

$R_{Z}^{2}=0.9974$

linear

$\left\{\begin{array}{l}X_{H D}=X_{0}+X_{q q} q^{2} \\ Z_{H D}=Z_{0}+Z_{q} q+Z_{q|q|} q|q| \\ M_{H D}=M_{0}+M_{q} q+M_{q|q|} q|q|\end{array}\right.$

$R_{M}^{2}=0.9997$

Linear

$$
\left\{\begin{array}{l}
X_{H D}=X_{0} \\
Y_{H D}=Y_{0}+Y_{p} p \\
K_{H D}=K_{0}+K_{p} p \\
N_{H D}=N_{0}+N_{p} p
\end{array}\right.
$$

$R_{Y}^{2}=0.9976$

$R_{K}^{2}=0.9999$

$R_{N}^{2}=0.8563$

Roll

rotating

Horizontal

Non-

linear

$$
\left\{\begin{array}{l}
X_{H D}=X_{0}+X_{p p} p^{2} \\
Y_{H D}=Y_{0}+Y_{p} p+Y_{p \mid p} p|p|
\end{array}\right.
$$

$R_{X}^{2}=0.9014$

$R_{Y}^{2}=0.9977$

$R_{K}^{2}=0.9997$

$R_{N}^{2}=0.8214$

$+X_{r r} r^{2}+X_{v r}$

$Y_{H D}=Y_{0}+Y_{v} v+Y_{v|v|} v|v|+Y_{r} r$

$R_{X}^{2}=0.9917$

circular

motion linear

with drift

$K_{H D}=K_{0}+K_{v} v+K_{v|v|} v \mid v+K_{r} r$

$+K_{r|r|} r|r|+K_{v|r|} v|r|+K_{|v| r}|v| r$

$N_{H D}=N_{0}+N_{v} v+N_{v|v|} v|v|+N_{r} r$
$\quad+N_{r i r}|r|+N$

$R_{Y}^{2}=0.9985$

$R_{K}^{2}=0.9528$

$R_{N}^{2}=0.9839$

$+N_{r|r|} r|r|+N_{v|r|} v|r|+N_{|v| r}|v| r$

Vertical

circular

motion

with aoa

$$
\text { Non- } \begin{cases}X_{H D}=X_{0}+X_{w w} w^{2}+X_{w w w w} w^{4} & \\ & +X_{q q} q^{2}+X_{w q} \\ Z_{H D}=Z_{0}+Z_{w} w+Z_{w|w|} w|w|+Z_{q} q & R_{X}^{2}=0.9796 \\ +Z_{q q} q^{2}+Z_{w \mid q}|w| q+Z_{w|q|} w|q| & R_{Z}^{2}=0.9961 \\ M_{H D}=M_{0}+M_{w} w+M_{w|w|} w|w|+ & R_{M}^{2}=0.9949 \\ M_{q} q+M_{q q} q^{2}+M_{w \mid q}|w| q+M_{w|q|} w|q| & \end{cases}
$$


approximately 1 . In addition, linear coefficients were identified within the drift angle range of $\pm 4^{\circ}$. As previously mentioned, hydrodynamic forces were calculated by adjusting the angle of attack by up to $\pm 90^{\circ}$, considering a large angle of attack; the results obtained from the static angle of attack test are presented in Fig. 6. A stall occurs at approximately $\pm 50^{\circ}$ and $\pm 40^{\circ}$ of the surge and pitch, respectively, and the tendency of forces should be modeled using the same model as the static drift test. Nonlinear coefficients constituting the nonlinear model must be analyzed using simple curve approximation results because physical implication is ambiguous. In contrast, linear coefficients comprising the linear model have a distinct physical implication, such that the effectiveness of numerical analysis results can be determined by examining the correlation among linear coefficients when there are
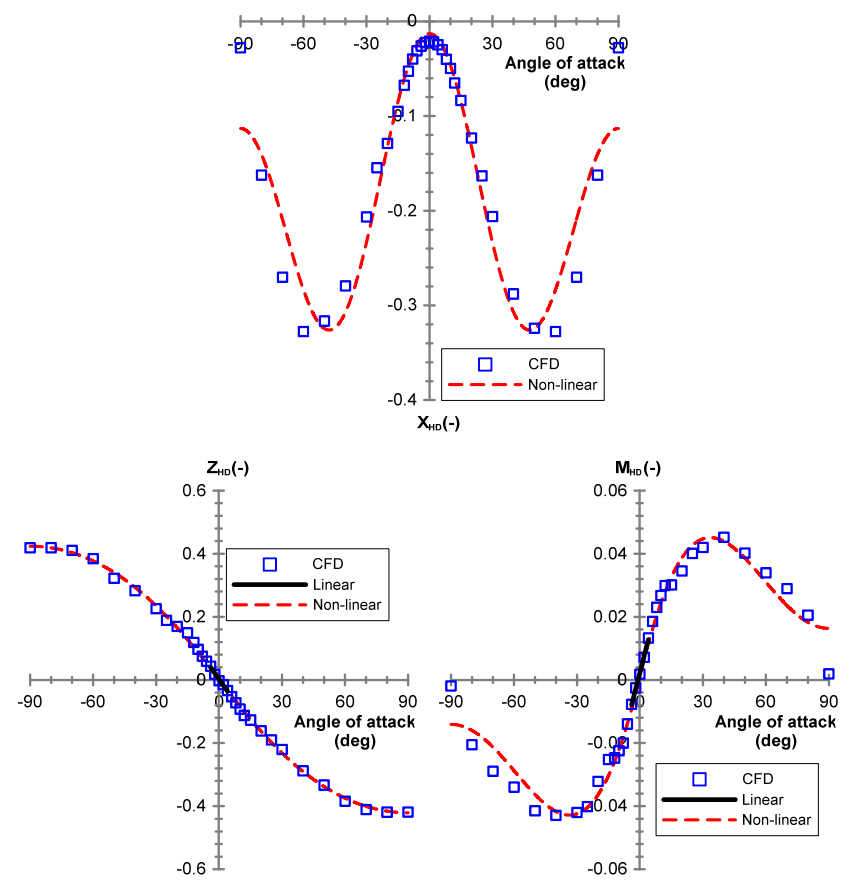

Fig. 6 Results obtained from static angle of attack tests
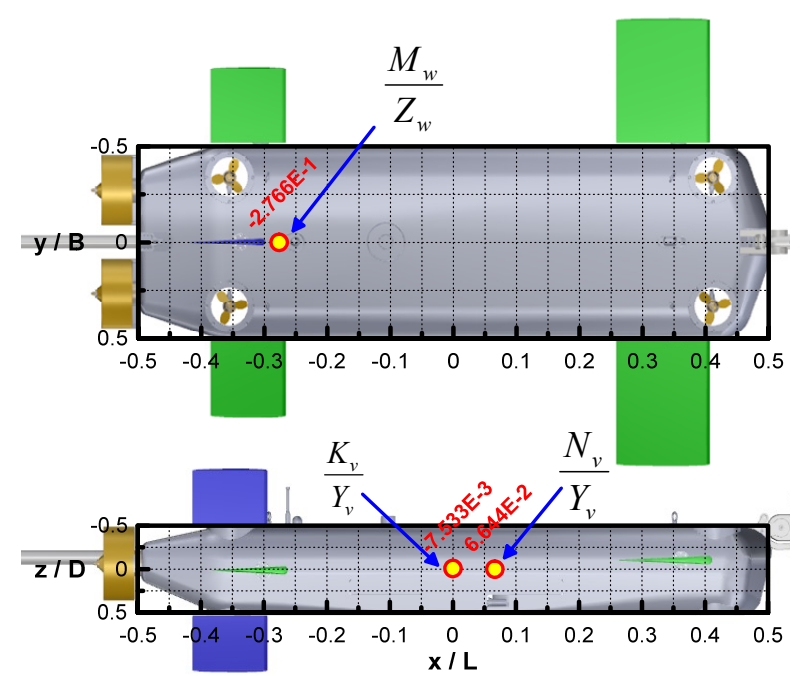

Fig. 7 Moment levers due to heave (upper) and sway (lower) velocities no experimental results, as in this study. Fig. 7 presents the points of damping force applications when the sway $v$ and heave $w$ velocities are generated based on the linear coefficients of stability $Y_{v}, K_{v}, N_{v}$, $Z_{w}$, and $M_{w}$, identified via the static drift and static angle of attack tests.

The side shape of the subject submerged body exhibits a tendency in which the rear part area is predominant owing to the large rudder attached in the rear part; thus, it is predicted to exhibit relatively better results in terms of horizontal stability. Therefore, $N_{v} / Y_{v}$, which is the application point of the damping force in the longitudinal direction due to the sway velocity, is positioned at approximately $0.066 \mathrm{~L}$ in front of the origin. Considering that the $N_{v} / Y_{v}$ of a general slender-type vehicle such as a ship is approximately $0.25 L$, the application point of the damping force moved backward considerably, toward the rear. Because the vertical shape is almost symmetrical, the application point $K_{v} / Y_{v}$ of the damping force in the height direction is positioned slightly upward with respect to the geometrical origin; however, the size is negligible. In contrast, $M_{w} / Z_{w}$, which is the application point of the damping force in the longitudinal direction due to the heave velocity, is positioned approximately $0.277 \mathrm{~L}$ toward the rear, with respect to the origin. A large bow plane exists in the head part, which implies that it is disadvantageous in terms of vertical stability, owing to the predominant head part.

Figs. 8 and 9 present the results obtained from horizontal and vertical turning tests, which are conducted to determine the damping force and moment related to the yaw angular velocity $r$ and pitch velocity $q$. The top area is more than two times greater than the side area of the hull, and the vertical hydrodynamic moment $M_{H D}$ is substantially greater than the horizontal hydrodynamic moment $Y_{H D}$. In contrast, hydrodynamic forces $Y_{H D}$ and $Z_{H D}$ are the forces generated from the difference in the shapes of the head and rear parts
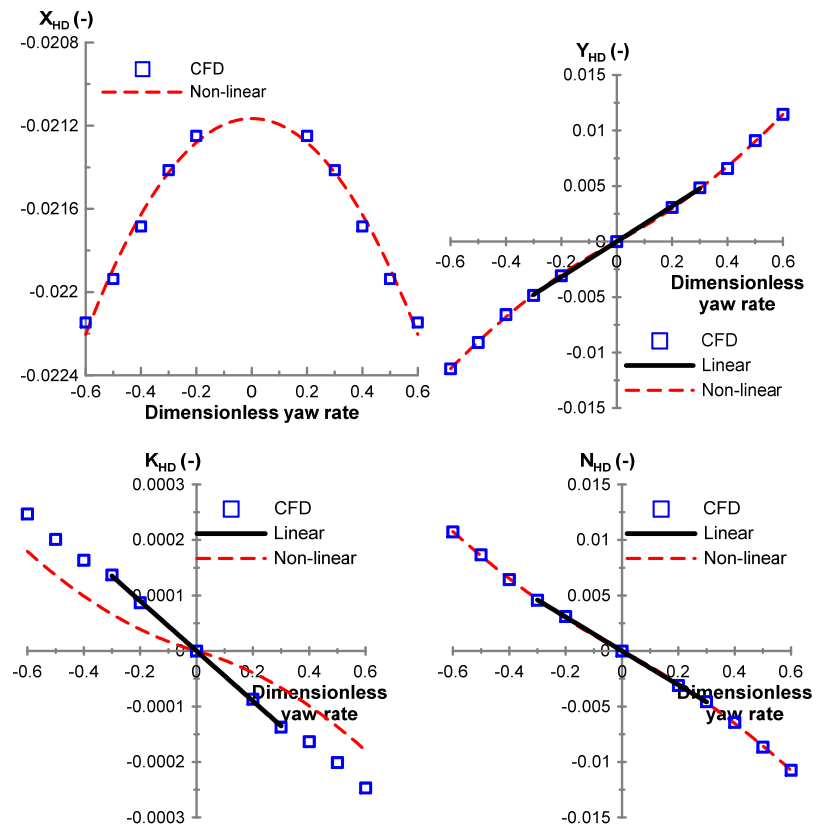

Fig. 8 Results obtained from horizontal circular motion tests 

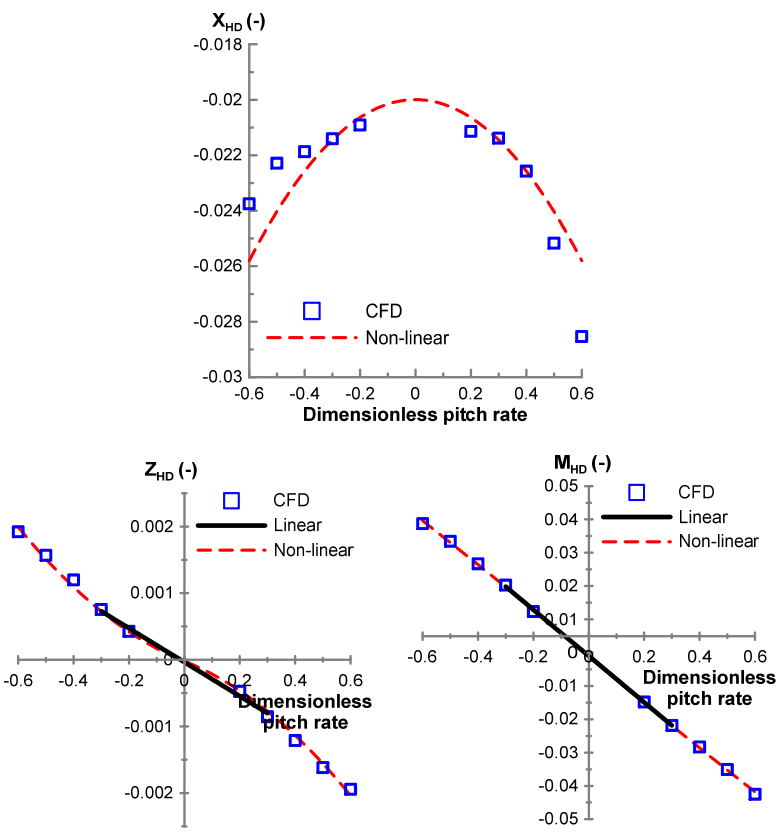

Fig. 9 Results obtained from vertical circular motion tests
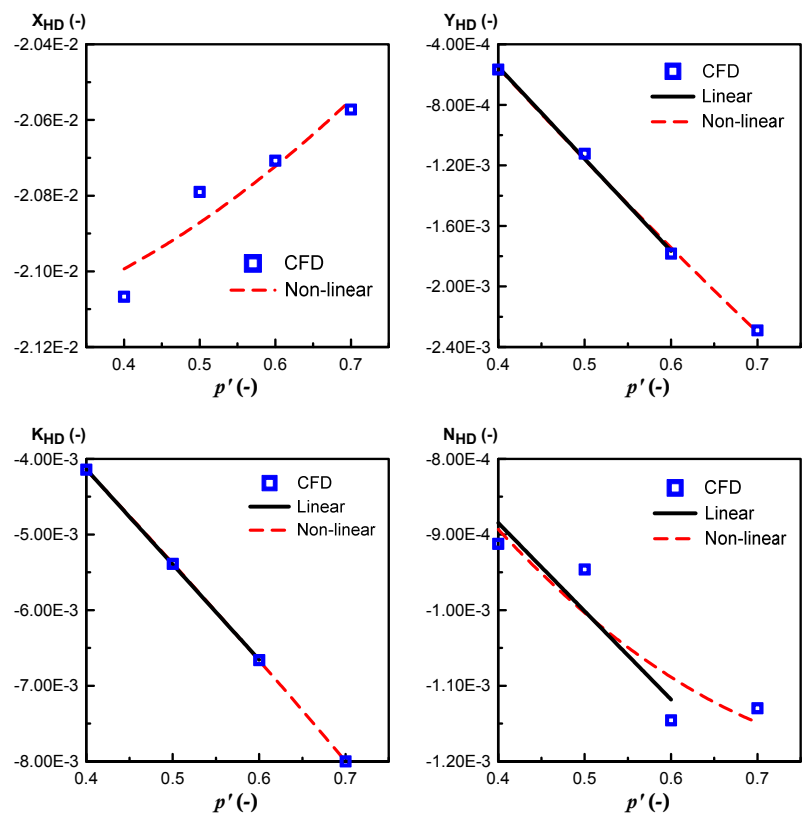

Fig. 10 Results obtained from roll rotating tests

during the rotational motions in which the asymmetry of the shapes of the head and rear parts significantly influence the horizontal hydrodynamic force $Y_{H D}$.

Fig. 10 presents the results of a roll rotating test, which measures the force generated by a certain roll rate $p$ while a submerged body is advanced at speed $U$. Generally, a submerged body with a cylindrical shape frequently experiences a large rolling motion if a controller is not applied because the roll damping coefficient applied on the hull is relatively small. The subject submerged body in this study has a relatively flat hull top surface and a large elevator area, thus exhibiting a large roll damping moment. As shown in Fig. 10, which illustrates the roll damping moment $K_{H D}$ for the roll rate $p$, the order is greater than that of the same angular motion moment $N_{H D}$. A large roll damping moment indicates that a roll is not large during circular motions in which favorable dynamic characteristics can be obtained from the motion control perspective. A simulation must be conducted to verify whether such a phenomenon actually occurs.

Figs. 11 and 12 present the results of the horizontal circular motion with the drift test and those of the vertical circular motion with the angle of attack test. The hydrodynamic forces measured in the static
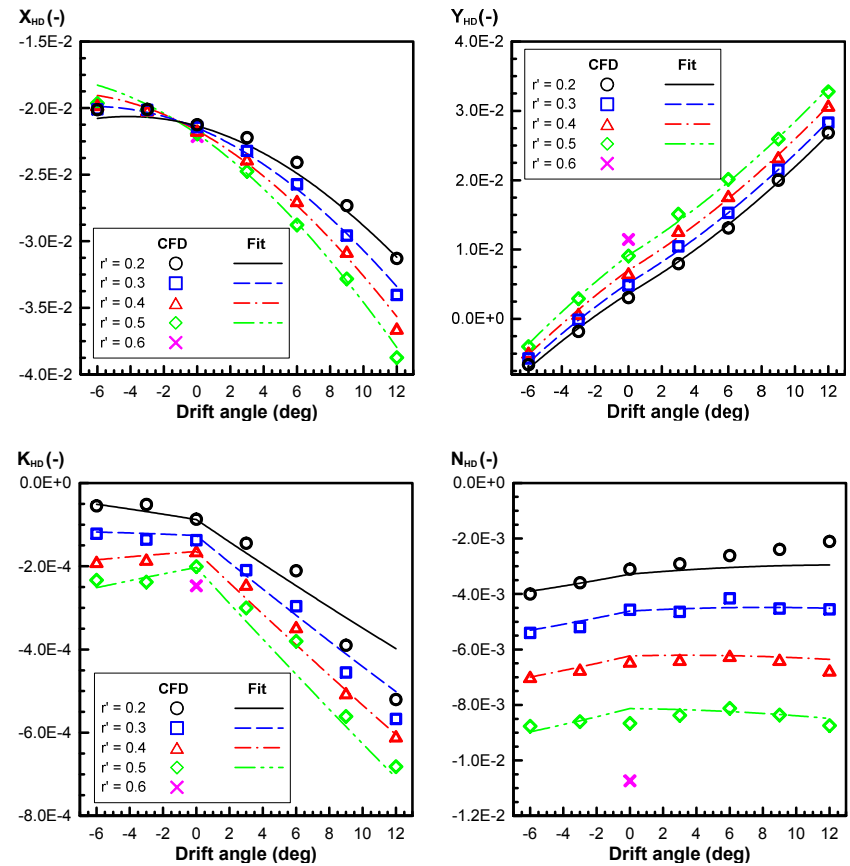

Fig. 11 Results obtained from the horizontal circular motion with drift tests
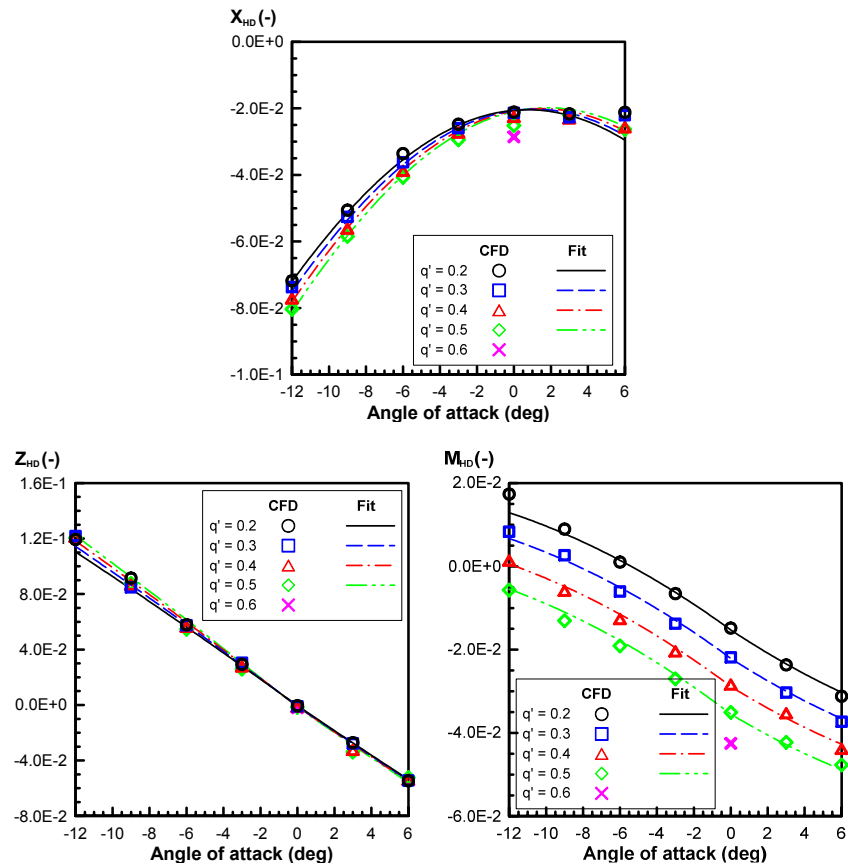

Fig. 12 Results obtained from the vertical circular motion with the angle of attack tests 
drift and horizontal turning tests are also measured when the horizontal circular motion with the drift test is performed; therefore, all hydrodynamic forces applied by and can be determined. Likewise, the hydrodynamic forces measured in the static angle of attack and vertical turning tests are also measured if the vertical circular motion is performed with drift test; therefore, all hydrodynamic forces applied by $w$ and $q$ can be determined.

All the damping coefficients due to ship motions $u, v, w, p, q$, and $r$ were estimated via the above tests. Table 5 presents the coefficient of determination of the hydrodynamic force model and corresponding models generated in the static rudder and static elevator tests. Figs. 1314 present the results of the static rudder and static elevator tests, respectively. In general, the center of pressure of the hydrodynamic forces applied on a rudder is positioned at approximately $1 / 4$ the point of a rudder chord. Similar to the static drift test, hydrodynamic forces and the rudder angle have a linear relationship in a region with a small rudder angle; hence, a linear coefficient can be determined to estimate the center of pressure of the rudder force. Fig. 15 presents the center of pressure on a rudder, which is estimated based on a linear coefficient when the rudder was rotated by a small angle. The center of pressure in the longitudinal direction $N_{\delta_{r}} / Y_{\delta_{r}}$ when the rudder was rotated is positioned closer to $1 / 4$ of a rudder chord. The center of pressure in the vertical direction $K_{\delta_{r}} / Y_{\delta_{r}}$ is slightly more predominant in the top area from the side view of the hull; however, the size of $K_{\delta_{r}} / Y_{\delta_{r}}$ is negligible because the vertical shape of the hull is almost symmetrical. The centers of pressure when the bow plane and stern elevator are turned, $M_{\delta_{b}} / Z_{\delta_{b}}$ and $M_{\delta_{s}} / Z_{\delta_{s}}$, are also approximate to the $1 / 4$ point of a

Table 5 Static hydrodynamic force model for control plane angles

\begin{tabular}{|c|c|c|c|}
\hline Test type & Model & Formulae & $R^{2}$ \\
\hline \multirow{2}{*}{$\begin{array}{l}\text { Static } \\
\text { rudder }\end{array}$} & Linear & $\left\{\begin{array}{l}X_{H D}=X_{0} \\
Y_{H D}=Y_{0}+Y_{\delta_{r}} \delta_{r} \\
K_{H D}=K_{0}+K_{\delta_{r}} \delta_{r}, \quad-4^{\circ}<\beta<4^{\circ} \\
N_{H D}=N_{0}+N_{\delta_{r}} \delta_{r}\end{array}\right.$ & $\begin{array}{l}R_{Y}^{2}=0.9981 \\
R_{K}^{2}=0.9517 \\
R_{N}^{2}=0.9995\end{array}$ \\
\hline & $\begin{array}{l}\text { Non- } \\
\text { linear }\end{array}$ & $\left\{\begin{array}{l}X_{H D}=X_{0}+X_{\delta_{,} \delta_{r}} \delta_{r}^{2} \\
Y_{H D}=Y_{0}+Y_{\delta_{r}} \delta_{r}+Y_{\delta_{r} \mid \delta_{r}}\left|\delta_{r}\right| \delta_{r} \mid \\
K_{H D}=K_{0}+K_{\delta_{r}} \delta_{r}+K_{\delta_{r}}\left|\delta_{r}\right| \delta_{r}\left|\delta_{r}\right| \\
N_{H D}=N_{0}+N_{\delta_{r}} \delta_{r}+N_{\delta_{r}\left|\delta_{r}\right|}\left|\delta_{r}\right| \delta_{r} \mid\end{array}\right.$ & $\begin{array}{l}R_{X}^{2}=0.9952 \\
R_{Y}^{2}=0.9992 \\
R_{K}^{2}=0.8317 \\
R_{N}^{2}=0.9857\end{array}$ \\
\hline \multirow{2}{*}{$\begin{array}{l}\text { Static } \\
\text { elevator } \\
\text { (Bow) }\end{array}$} & Linear & $\left\{\begin{array}{l}X_{H D}=X_{0} \\
Z_{H D}=Z_{0}+Z_{\delta_{b}} \delta_{b} \\
M_{H D}=M_{0}+M_{\delta_{b}} \delta_{b}\end{array}\right.$ & $\begin{array}{l}R_{Z}^{2}=0.9991 \\
R_{M}^{2}=0.9983\end{array}$ \\
\hline & $\begin{array}{l}\text { Non- } \\
\text { linear }\end{array}$ & $\left\{\begin{array}{l}X_{H D}=X_{0}+X_{\delta_{b} \mid \delta_{b}}\left|\delta_{b}\right| \delta_{b} \mid \\
Z_{H D}=Z_{0}+Z_{\delta_{b}} \delta_{b}+Z_{\delta_{b}}\left|\delta_{b}\right| \delta_{b}\left|\delta_{b}\right| \\
M_{H D}=M_{0}+M_{\delta_{b}} \delta_{b}+M_{\delta_{b}}\left|\delta_{b}\right| \delta_{b}\left|\delta_{b}\right|\end{array}\right.$ & $\begin{array}{l}R_{X}^{2}=0.9828 \\
R_{Z}^{2}=0.9943 \\
R_{M}^{2}=0.9984\end{array}$ \\
\hline \multirow{2}{*}{$\begin{array}{l}\text { Static } \\
\text { elevator } \\
\text { (Stern) }\end{array}$} & Linear & $\left\{\begin{array}{l}X_{H D}=X_{0} \\
Z_{H D}=Z_{0}+Z_{\delta_{b}} \delta_{b} \\
M_{H D}=M_{0}+M_{\delta_{b}} \delta_{b}\end{array}\right.$ & $\begin{array}{l}R_{Z}^{2}=0.9509 \\
R_{M}^{2}=0.9177\end{array}$ \\
\hline & $\begin{array}{l}\text { Non- } \\
\text { linear }\end{array}$ & $\left\{\begin{array}{l}X_{H D}=X_{0}+X_{\delta_{s} \mid \delta_{s}} \delta_{s}\left|\delta_{s}\right| \\
Z_{H D}=Z_{0}+Z_{\delta_{s}} \delta_{s}+Z_{\delta_{s}\left|\delta_{s}\right|} \delta_{s}\left|\delta_{s}\right| \\
M_{H D}=M_{0}+M_{\delta_{s}} \delta_{s}+M_{\delta_{s}}\left|\delta_{s}\right| \delta_{s}\left|\delta_{s}\right|\end{array}\right.$ & $\begin{array}{l}R_{X}^{2}=0.9739 \\
R_{Z}^{2}=0.9944 \\
R_{M}^{2}=0.9917\end{array}$ \\
\hline
\end{tabular}
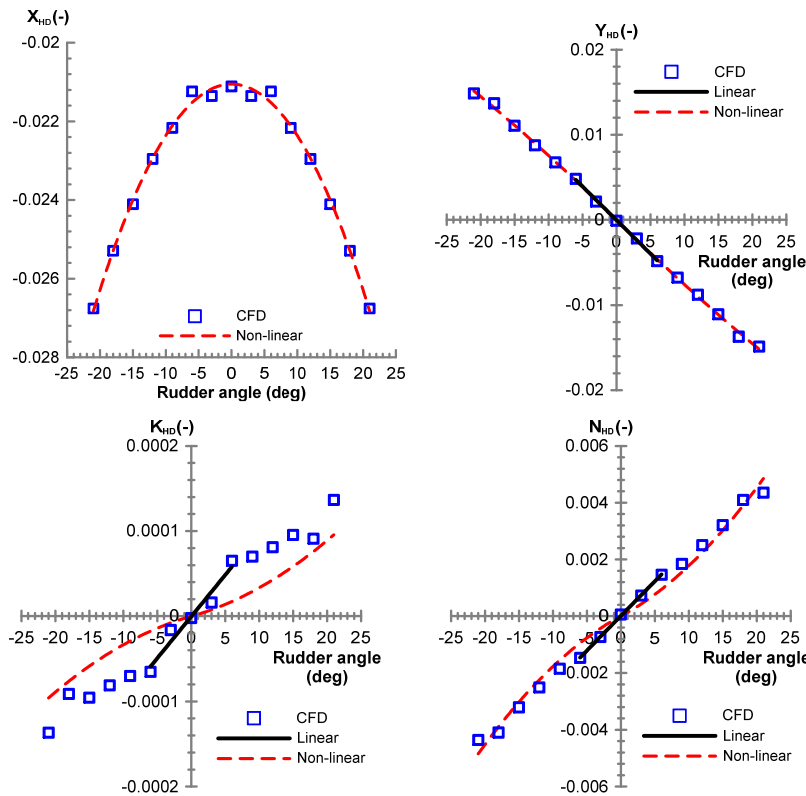

Fig. 13 Results obtained from static rudder tests
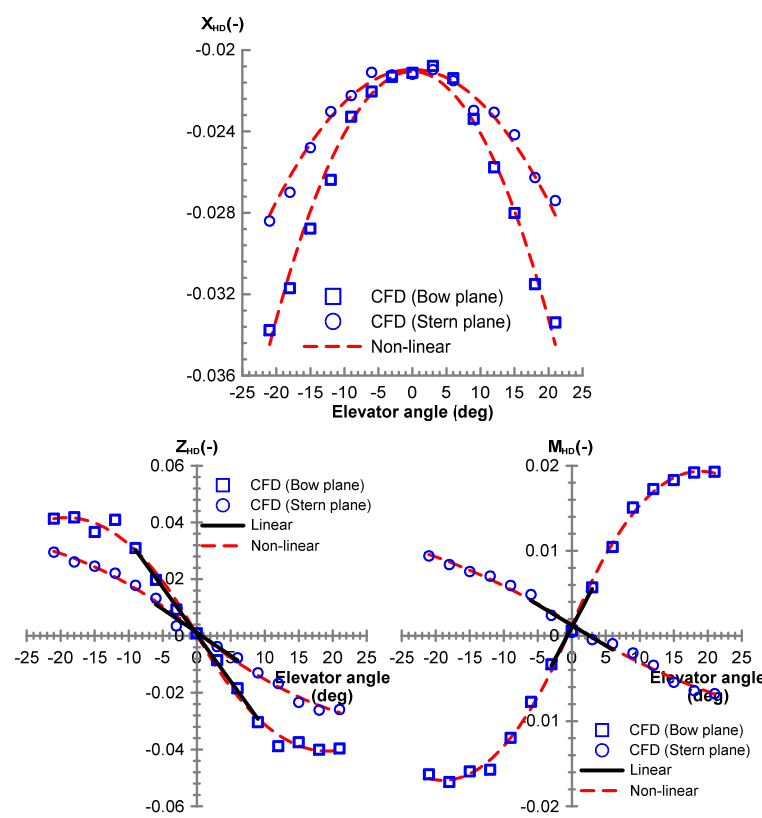

Fig. 14 Results obtained from static elevator tests

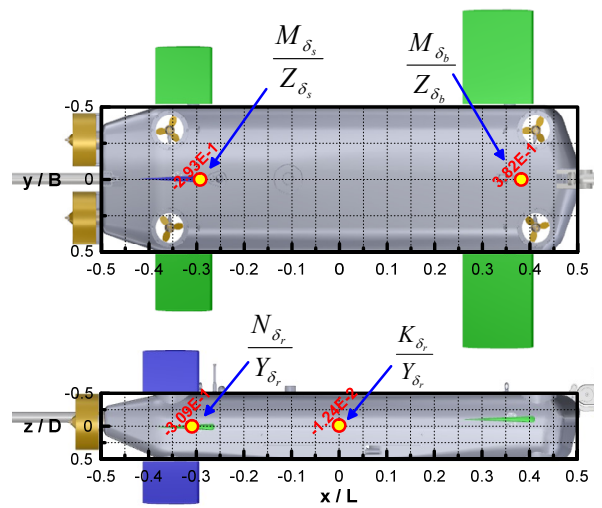

Fig. 15 Moment levers due to elevator (upper) and rudder (lower) deflections 
Table 6 Dimensionless linear maneuvering mathematical model parameters $\left(\times 10^{6}\right)$

\begin{tabular}{cccccc}
\hline \multicolumn{2}{c}{$X$-coeff. } & \multicolumn{2}{c}{$Y$-coeff. } & \multicolumn{2}{c}{$Z$-coeff. } \\
\hline$X_{\dot{u}}$ & -1872 & $Y_{\dot{v}}$ & -59564 & $Z_{\dot{w}}$ & -299190 \\
$X_{u}$ & -21472 & $Y_{\dot{p}}$ & 54 & $Z_{\dot{q}}$ & -65503 \\
& & $Y_{\dot{r}}$ & 29365 & $Z_{w}$ & -535933 \\
& & $Y_{v}$ & -89962 & $Z_{q}$ & -2548 \\
& & $Y_{p}$ & -6083 & $Z_{\delta_{b}}$ & -199688 \\
& & $Y_{r}$ & 15920 & $Z_{\delta_{s}}$ & -94044 \\
& & $Y_{\delta_{r}}$ & -45209 & & \\
\hline$K_{\dot{v}}$ & -758 & $M_{\dot{w}}$ & 71802 & $N_{\dot{v}}$ & 2282 \\
$K_{\dot{p}}$ & -1004 & $M_{\dot{q}}$ & -39941 & $N_{\dot{p}}$ & -34 \\
$K_{\dot{r}}$ & -490 & $M_{w}$ & 148263 & $N_{\dot{r}}$ & -10956 \\
$K_{v}$ & 678 & $M_{q}$ & -69468 & $N_{v}$ & -5977 \\
$K_{p}$ & -12587 & $M_{\delta_{b}}$ & 76212 & $N_{p}$ & -1167 \\
$K_{r}$ & -450 & $M_{\delta_{s}}$ & -27535 & $N_{r}$ & -15323 \\
$K_{\delta_{r}}$ & 560 & & & $N_{\delta_{r}}$ & 13978 \\
\hline
\end{tabular}

rudder chord. Fig. 14 presents the results obtained from comparing the hydrodynamic forces when the bow plane was turned and when the stern elevator was turned. The area of a bow plane is approximately twice as large as the area of a stern elevator; hence, the linear control panel coefficient $Z_{\delta_{b}}$ provided in Table 6 is approximately twice as large as $Z_{\delta_{s}}$. In contrast, moment coefficients $M_{\delta_{b}}$ and $M_{\delta_{s}}$ are associated with the distance to the pressure center of a control panel, and $M_{\delta_{b}}$ is at least two times greater than $M_{\delta_{s}}$ because the pressure center of a bow plane is far from the origin. Similarly, examining the physical relationship between linear coefficients appears to be an appropriate method for verifying the CFD analysis results when no experimental results are available.

\subsection{Dynamic Test}

A dynamic test was performed to estimate the added mass force applied on the hull and the surrounding fluid when the hull accelerates. The added mass force exhibits a linear relationship with acceleration when a vessel or a submerged body has a very small accelerated motion. Figs. 16 and 17 present the results obtained from pure sway tests for estimating the added mass force with respect to sway acceleration $\dot{v}$ and pure heave tests for estimating the added mass force with respect to heave acceleration $\dot{w}$, respectively. The added mass moment of inertia coefficients $K_{\dot{v}}$ and $N_{\dot{v}}$ for $\dot{v}$ are typically negligible, as well as the added mass moment of inertia coefficient $M_{w}$ for $\dot{w}$; therefore, the sizes of $Y_{\dot{v}}$ and $Z_{\dot{w}}$ need to be examined. The results obtained from comparing the sizes of $Y_{\dot{v}}$ and $Z_{\dot{w}}$ with the mass of the submerged body are illustrated in Fig. 18. $Y_{\dot{v}}$ of a general ship has proportion of its mass. Furthermore, $Y_{\dot{v}}$ and $Z_{\dot{w}}$ are identical in a
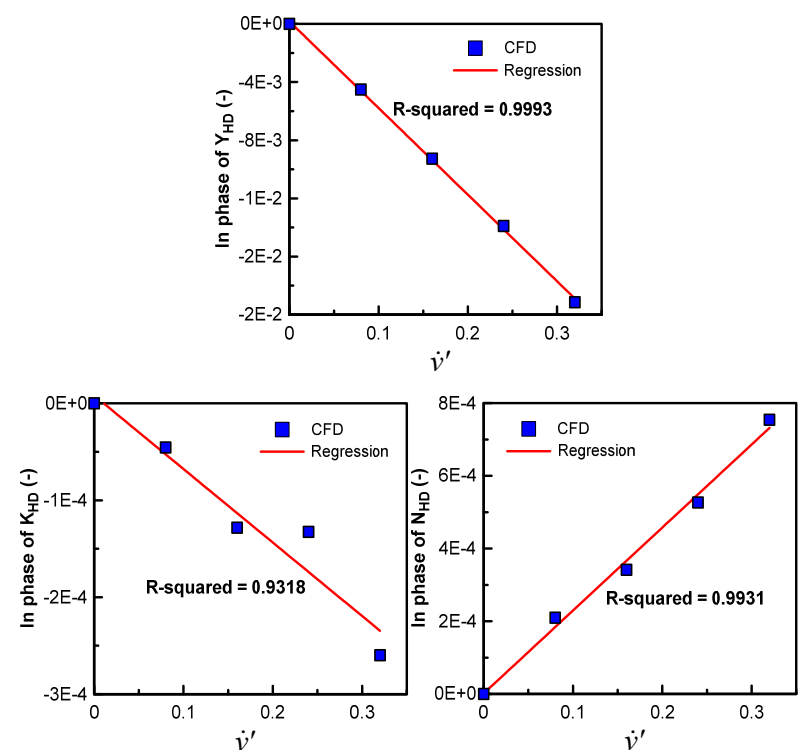

Fig. 16 Results obtained from pure sway tests
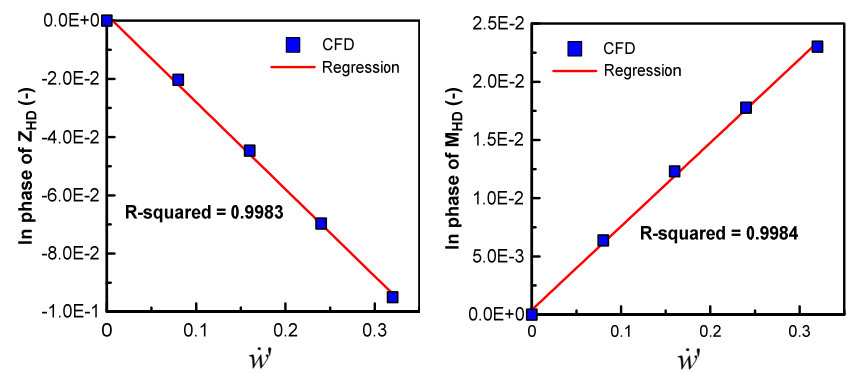

Fig. 17 Results obtained from pure heave tests

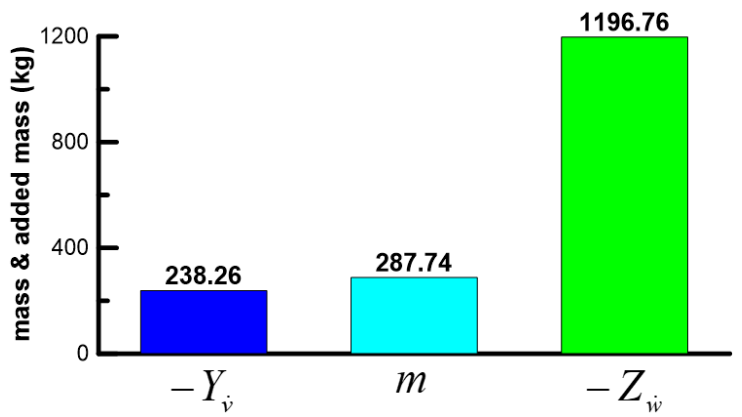

Fig. 18 Comparison of mass and added mass

submerged body with symmetrical horizontal and vertical planes. However, the subject submerged body in this study is applied with a relatively greater added mass force owing to its plate shape. Considering that the top surface area is at least twice as large as the side area and the top surface shape is a plate shape, rather than a streamlined shape, it is feasible for the size of $Z_{w}$ to be greater than that of $Y_{i}$ and its own mass.

Figs. 19-21 illustrate the results obtained from a dynamic test for angular acceleration. Figs. 19 and 20 present the results obtained from estimating added mass coefficients for yaw angular acceleration $\dot{r}$ and pitch angular acceleration $\dot{q}$. Similar to the difference in the sizes of $Y$, and $Z_{\dot{w}}$, the size of $M_{q}$ is greater than that of $N_{\dot{r}}$ because the top 

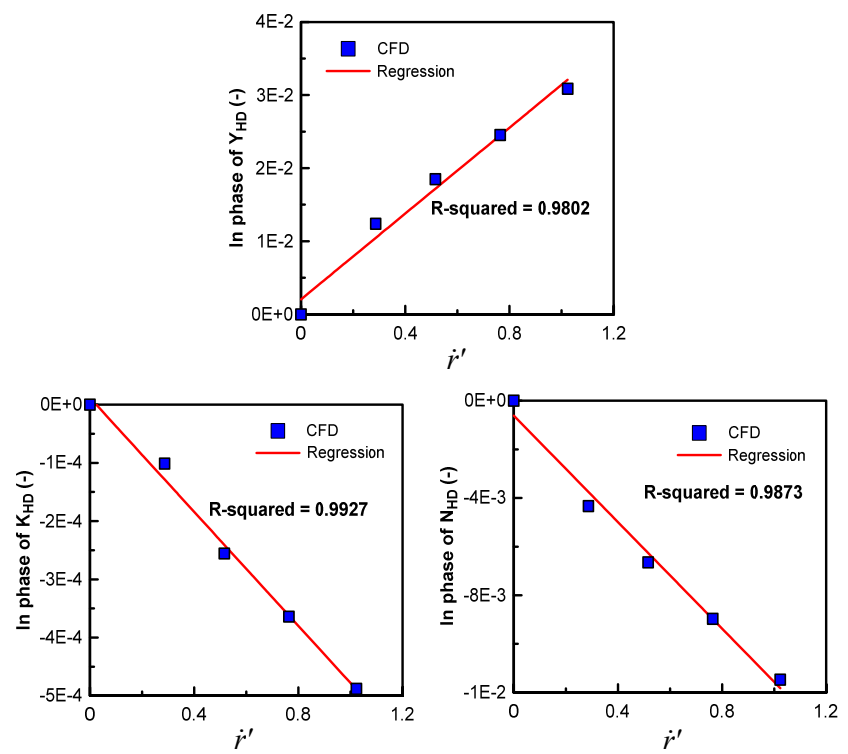

Fig. 19 Results obtained from pure yaw tests
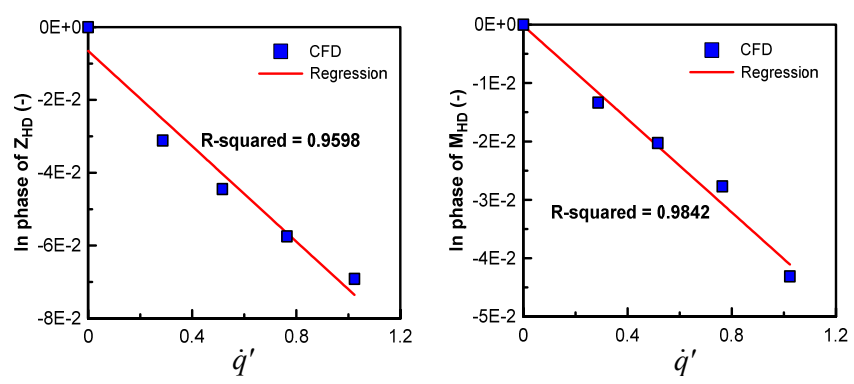

Fig. 20 Results obtained from pure pitch tests
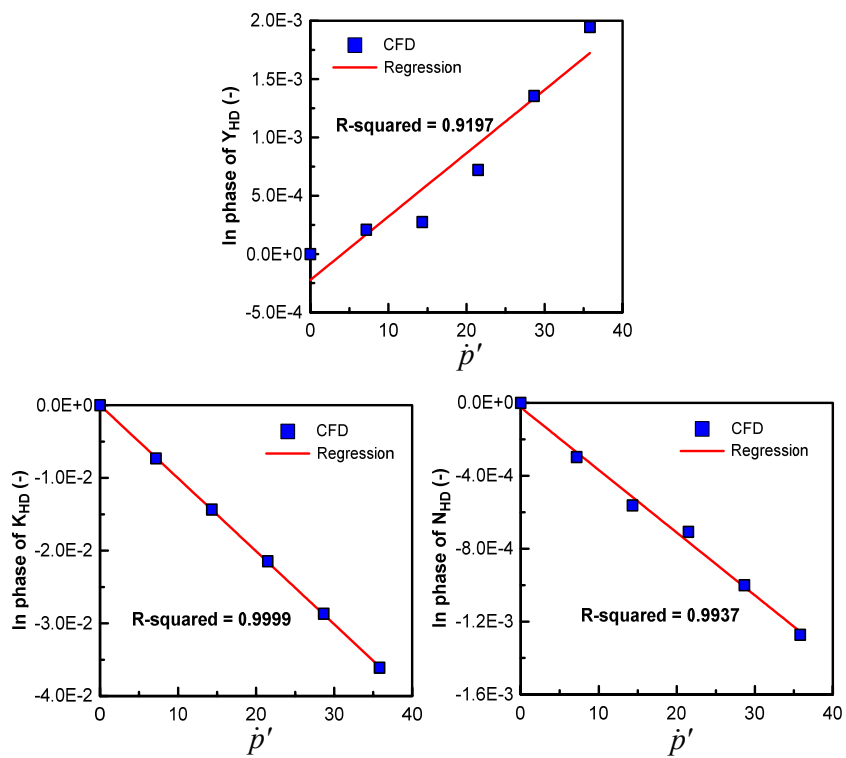

Fig. 21 Results obtained from pure roll tests

surface shape is more predominant than that of the side shape. In general, the added mass moment of inertia is sufficiently small to be negligible compared to the mass moment of inertia $\left(I_{x x}\right)$ in a submerged body with a streamlined shape when a roll angular acceleration $\dot{p}$ is generated. However, the subject submerged body has a relatively plate-like shape, and the slenderness ratio $(L / B)$ is relatively small. Moreover, a large hydrodynamic moment is generated by a roll angular acceleration because the areas of bow and stern elevators are large. Consequently, the yaw induced mass moment of inertia $K_{p}$ is relatively larger than that of a general slender-type submerged body, as illustrated in Fig. 21.

\subsection{Dynamics Model}

The six degrees-of-freedom equations of motion based on Newton's second law of motion can be expressed as in Eq. (1).

$$
\left\{\begin{array}{l}
m\left[\dot{u}-v r+w q-x_{g}\left(q^{2}+r^{2}\right)+z_{g}(p r+\dot{q})\right]=X \\
m\left[\dot{v}-w p+u r+z_{g}(q r-\dot{p})+x_{g}(q p+\dot{r})\right]=Y \\
m\left[\dot{w}-u q+v p-z_{g}\left(r^{2}+p^{2}\right)+x_{g}(r p-\dot{q})\right]=Z \\
I_{x} \dot{p}+\left(I_{z}-I_{y}\right) q r-m\left[z_{g}(\dot{v}-w p+u r)\right]=K \\
I_{y} \dot{q}+\left(I_{x}-I_{z}\right) r p+m\left[z_{g}(\dot{u}-v r+w q)-x_{g}(\dot{w}-u q+v p)\right]=M \\
I_{z} \dot{r}+\left(I_{y}-I_{x}\right) p q+m\left[x_{g}(\dot{v}-w p+u r)\right]=N
\end{array}\right.
$$

External forces on the right side of Eq. (1) can be divided into hydrodynamic force, gravitational force, buoyant force, control force, and thrust. Hydrodynamic forces were modeled as expressed in Eqs. (2) and (3) by distinguishing linear models for designing the controller and stability analysis and nonlinear models for predicting maneuverability based on the coefficients identified via the static and dynamic tests.

$$
\begin{aligned}
& \left\{\begin{array}{l}
X_{H D}=X_{\dot{u}} \dot{u}+X_{u} u \\
Y_{H D}=Y_{\dot{v}} \dot{v}+Y_{\dot{p}} \dot{p}+Y_{\dot{r}} \dot{r}+Y_{v} v+Y_{p} p+Y_{r} r
\end{array}\right. \\
& \left\{Z_{H D}=Z_{\dot{w}} \dot{w}+Z_{\dot{q}} \dot{q}+Z_{w} w+Z_{q} q\right. \\
& K_{H D}=K_{\dot{v}} \dot{v}+K_{\dot{p}} \dot{p}+K_{\dot{r}} \dot{r}+K_{v} v+K_{p} p+K_{r} r \\
& M_{H D}=M_{\dot{w}} \dot{w}+M_{\dot{q}} \dot{q}+M_{w} w+M_{q} q \\
& N_{H D}=N_{\dot{v}} \dot{v}+N_{\dot{p}} \dot{p}+N_{\dot{r}} \dot{r}+N_{v} v+N_{p} p+N_{r} r \\
& \left\{\begin{aligned}
X_{H D} & =X_{\dot{u}} \dot{u}+X_{u|u|} u|u|+X_{v v} v^{2}+X_{v v v v} v^{4}+X_{w w} w^{2}+X_{w w w w} w^{4} \\
& +X_{p p} p^{2}+X_{q q} q^{2}+X_{r r} r^{2}+X_{v r} v r+X_{w q} w q \\
Y_{H D} & =Y_{\dot{v}} \dot{v}+Y_{\dot{p}} \dot{p}+Y_{\dot{r}} \dot{r}+Y_{v} v+Y_{p} p+Y_{r} r \\
& +Y_{v|v|} v|v|+Y_{p|p|} p|p|+Y_{r|r|} r|r|+Y_{v|r|} v|r|+Y_{|v| r}|v| r \\
Z_{H D} & =Z_{\dot{w}} \dot{w}+Z_{\dot{q}} \dot{q}+Z_{*}+Z_{w} w+Z_{q} q \\
& +Z_{w|w|} w|w|+Z_{q|q|}\left|q+Z_{w|q|} w\right| q\left|+Z_{|w| q}\right| w \mid q
\end{aligned}\right. \\
& \left\{\begin{aligned}
K_{H D} & =K_{\dot{v}} \dot{v}+K_{\dot{p}} \dot{p}+K_{\dot{r}} \dot{r}+K_{v} v+K_{p} p+K_{r} r \\
& +K_{v|v|} v|v|+K_{p \mid p}|p|+K_{r|r|} r|r|+K_{v|r|} v|r|+K_{|v| r}|v| r
\end{aligned}\right. \\
& M_{H D}=M_{\dot{w}} \dot{w}+M_{q} \dot{q}+Z_{*}+M_{w} w+M_{q} q \\
& +M_{w|w|}^{w} w|w|+M_{q|q|} q\left|q+M_{w|q|} w\right| q\left|+M_{w \mid q}\right| w \mid q \\
& N_{H D}=N_{\dot{v}} \dot{v}+N_{\dot{p}} \dot{p}+N_{\dot{r}} \dot{r}+N_{v} v+N_{p} p+N_{r} r \\
& +N_{v|v|} v|v|+N_{p|p|} p|p|+N_{r|r|} r|r|+N_{v|r|} v|r|+N_{|v| r}|v| r
\end{aligned}
$$

$$
\left\{\begin{array}{l}
X_{C}=0 \\
Y_{C}=Y_{\delta_{r}} \delta_{r} \\
Z_{C}=Z_{\delta_{b}} \delta_{b}+Z_{\delta_{s}} \delta_{s} \\
K_{C}=K_{\delta_{r}} \delta_{r} \\
M_{C}=M_{\delta_{b}} \delta_{b}+M_{\delta_{s}} \delta_{s} \\
N_{C}=N_{\delta_{r}} \delta_{r}
\end{array}\right.
$$




$$
\left\{\begin{array}{l}
X_{C}=X_{\delta_{r} \delta_{r}} \delta_{r}^{2} \\
Y_{C}=Y_{\delta_{r}} \delta_{r}+Y_{\delta_{r}}\left|\delta_{r}\right| \delta_{r}\left|\delta_{r}\right| \\
Z_{C}=Z_{\delta_{b}} \delta_{b}+Z_{\delta_{s}} \delta_{s}+Z_{\delta_{b}}\left|\delta_{b}\right| \delta_{b}\left|\delta_{b}\right|+Z_{\delta_{s}}\left|\delta_{s}\right| \delta_{s}\left|\delta_{s}\right| \\
K_{C}=K_{\delta_{r}} \delta_{r}+K_{\delta_{r}\left|\delta_{r}\right|}\left|\delta_{r}\right| \delta_{r} \mid \\
M_{C}=M_{\delta_{b}} \delta_{b}+M_{\delta_{s}} \delta_{s}+M_{\delta_{b}}\left|\delta_{b}\right| \delta_{b}\left|\delta_{b}\right|+M_{\delta_{s}}\left|\delta_{s}\right| \delta_{s}\left|\delta_{s}\right| \\
N_{C}=N_{\delta_{r}} \delta_{r}+N_{\delta_{r}\left|\delta_{r}\right|}\left|\delta_{r}\right| \delta_{r} \mid
\end{array}\right.
$$

Unlike other external forces contributing to the surface force, gravitational force and buoyancy are body forces that contribute to the volume, which do not require a modeling process as hydrodynamic forces, and can be perfectly expressed physically. Therefore, the expressions for gravitational force and buoyancy are omitted in this study because they can be referenced in the study by Fossen (2011). A control force is a fluid force applied on the control panel and the hull when the control panel rotates. Eqs. (4) and (5) represent the control force of linear and nonlinear models, respectively. The parameters expressed in Eqs. (2)-(5) are presented in Tables 6 and 7 by distinguishing between a linear and nonlinear model.

Table 7 Dimensionless non-linear maneuvering mathematical model parameters $\left(\times 10^{6}\right)$

\begin{tabular}{cccccc}
\hline \multicolumn{2}{c}{$X$-coeff. } & \multicolumn{2}{c}{$Y$-coeff. } & \multicolumn{2}{c}{$Z$-coeff. } \\
\hline$X_{\dot{u}}$ & -1872 & $Y_{\dot{v}}$ & -59564 & $Z_{\dot{w}}$ & -299190 \\
$X_{u|u|}$ & -43077 & $Y_{\dot{p}}$ & 54 & $Z_{\dot{q}}$ & -65503 \\
$X_{v v}$ & -180760 & $Y_{\dot{r}}$ & 29365 & $Z_{*}$ & 1474 \\
$X_{v v v v}$ & 113190 & $Y_{v}$ & -75834 & $Z_{w}$ & -510550 \\
$X_{w w}$ & -1142600 & $Y_{v|v|}$ & -146670 & $Z_{w|w|}$ & 88318 \\
$X_{w w w w}$ & 1042500 & $Y_{p}$ & -7178 & $Z_{q}$ & -1664 \\
$X_{p p}$ & 1354 & $Y_{p|p|}$ & 1224 & $Z_{q|q|}$ & -2861 \\
$X_{r r}$ & -2883 & $Y_{r}$ & 6663 & $Z_{w|q|}$ & -119730 \\
$X_{v r}$ & 9883 & $Y_{r|r|}$ & 16754 & $Z_{w \mid q}$ & 85992 \\
$X_{w q}$ & 140360 & $Y_{v|r|}$ & -40930 & $Z_{\delta_{b}}$ & -246238 \\
$X_{q q}$ & -1664 & $Y_{|v| r}$ & -21802 & $Z_{\delta_{b}\left|\delta_{b}\right|}$ & 368611 \\
$X_{\delta_{r} \delta_{r}}$ & -42934 & $Y_{\delta_{r}}$ & -45216 & $Z_{\delta_{s}}$ & -112358 \\
$X_{\delta_{b} \delta_{b}}$ & -99899 & $Y_{\delta_{r}\left|\delta_{r}\right|}$ & 10537 & $Z_{\delta_{s}\left|\delta_{s}\right|}$ & 95154 \\
$X_{\delta_{s} \delta_{s}}$ & -53328 & \multicolumn{3}{c}{} & \\
\hline \multicolumn{2}{c}{$K$-coeff. } & \multicolumn{2}{c}{$M$-coeff. } & $N_{\delta_{r}\left|\delta_{r}\right|}$ & 16017 \\
\hline$K_{\dot{v}}$ & -758 & $M_{\dot{w}}$ & 71802 & $N_{\dot{v}}$ & 2282 \\
$K_{\dot{p}}$ & -1004 & $M_{q}$ & -39941 & $N_{\dot{p}}$ & -34 \\
$K_{\dot{r}}$ & -490 & $M_{*}$ & 1136 & $N_{\dot{r}}$ & -10956 \\
$K_{v}$ & 926 & $M_{w}$ & 119002 & $N_{v}$ & -5191 \\
$K_{p}$ & -283 & $M_{w|w|}$ & -76339 & $N_{p}$ & 4703 \\
$K_{r}$ & -10230 & $M_{q}$ & 4713 & $N_{r}$ & -2223 \\
$K_{v|v|}$ & -2376 & $M_{q|q|}$ & 11909 & $N_{v|v|}$ & 1247 \\
$K_{p|p|}$ & -384 & $M_{w|q|}$ & 41481 & $N_{p|p|}$ & -6188 \\
$K_{r|r|}$ & 0 & $M_{\delta_{b}}$ & 110107 & $N_{r|r|}$ & -14236 \\
$K_{v|r|}$ & 200 & $M_{\delta_{b}\left|\delta_{b}\right|}$ & -167012 & $N_{v|r|}$ & 1989 \\
$K_{|v| r}$ & -2923 & $M_{\delta_{s}}$ & -29035 & $N_{|v| r}$ & -9039 \\
$K_{\delta_{r}}$ & 128 & $M_{\delta_{s}\left|\delta_{s}\right|}$ & 18252 & $N_{\delta_{r}}$ & 7364 \\
$K_{\delta_{r}\left|\delta_{r}\right|}$ & 363 & & & & \\
\hline & & & & & \\
\hline
\end{tabular}

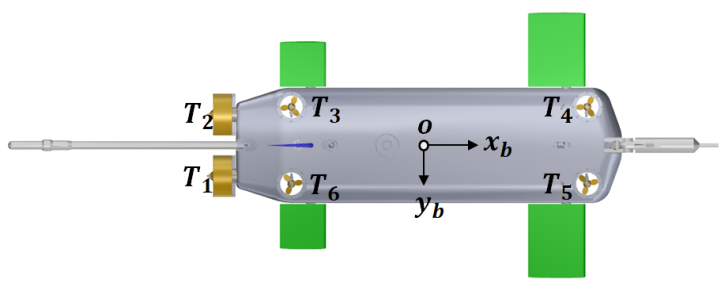

Fig. 22 Sequence of thruster

Because the system has six thrusters attached, the subjectsubmerged body can create thrust in all directions, except for the sway direction. Thrust force with six degrees of freedom generated by each thruster can be defined as expressed in Eq. (6), based on Fig. 22.

$$
\left\{\begin{array}{l}
X_{T}=T_{1}+T_{2} \\
Y_{T}=0 \\
Z_{T}=T_{3}+T_{4}+T_{5}+T_{6} \\
K_{T}=y_{T_{3}} T_{3}+y_{T_{4}} T_{4}+y_{T_{5}} T_{5}+y_{T_{6}} T_{6} \\
M_{T}=z_{T_{1}} T_{1}+z_{T_{2}} T_{2}-\left(x_{T_{3}} T_{3}+x_{T_{4}} T_{4}+x_{T_{5}} T_{5}+x_{T_{6}} T_{6}\right) \\
N_{T}=-\left(y_{T_{1}} T_{1}+y_{T_{2}} T_{2}\right)
\end{array}\right.
$$

$x_{T_{i}}, y_{T_{i}}$, and $z_{T_{i}}$ in Eq. (6) represent the distance from the origin of the $i$-th thruster to the $x$-, $y$-, $z$-axes, respectively. The thrust specified in the specifications of each thrust manufacturer was adopted as the thrust defined by $T_{1-6}$.

\section{Dynamics Simulation}

\subsection{Stability Analysis}

A stability analysis was conducted to evaluate the stability relative to the ship's course and depth while the control panel is fixed $\left(\delta_{r}=\delta_{b}=\delta_{s}=0\right)$. The sizes of the perturbation state variables $v, w, q$, and $r$ were not significant when the control panel was fixed and the linearization of the equations of motion in Eq. (1) was possible; hence, the linear stability coefficients in Table 6 were adopted. Horizontal and vertical stability can be evaluated based on the stability margin, under the assumption that surge velocity $u$ has no influence on sway, yaw, heave, or pitch. Horizontal and vertical stability margins can be defined by adopting Eqs. (7) and (8), respectively, at infinite speed.

$$
\begin{aligned}
& G_{h}=1-\frac{N_{v}\left(Y_{r}-m\right)}{Y_{v}\left(N_{r}-m x_{g}\right)} \\
& G_{v}=1-\frac{M_{v}\left(Z_{q}+m\right)}{Z_{w}\left(M_{q}-m x_{g}\right)}
\end{aligned}
$$

Because $m$ and $x_{g}$ in Eqs. (7)-(8) are design variables, the stability margin results in Fig. 23 can be deduced for $x_{g}$ under the assumption that neutral buoyancy is applied. As mentioned in Section 3, the rear is more critical with regard to the side shape of the submerged body, where it was assumed to be more important than the vertical plane in terms of stability. The analysis results indicated that $G_{h}$ is greater than $G_{v}$ when the center of gravity is closer to zero. 


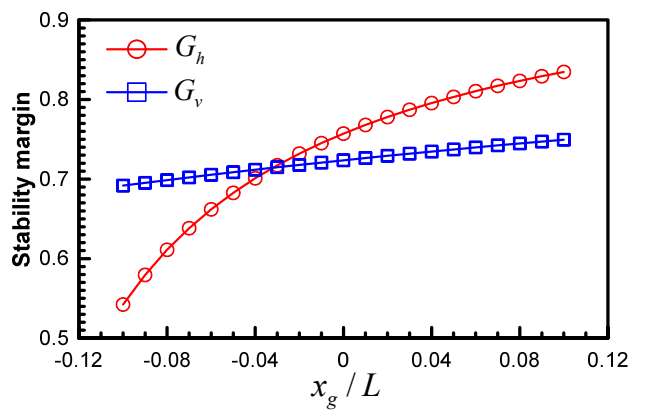

Fig. 23 Results obtained from pure roll tests

\subsection{Maneuverability Analysis}

Unlike a stability analysis in which the control panel is fixed, the control panel can be adjusted to the maximum angle when analyzing maneuverability, such as in turning and veering, which creates large motions such as drifting or yaw rate. Therefore, the hydrodynamic and control forces defined in Eqs. (3) and (5) are required. A turning simulation was performed by altering the rudder angle by $\pm 5^{\circ}, \pm 10^{\circ}$, and $\pm 15^{\circ}$ under an initial speed of $2.57 \mathrm{~m} / \mathrm{s}$, and the obtained results are illustrated in Figs. 24 and 25. The simulation results indicate that the tactical diameter is approximately $7.42 \mathrm{~m}$ when the rudder angle is $15^{\circ}$. The hull length of the subject submerged body is $2 \mathrm{~m}$, and the entire length is approximately $3.7 \mathrm{~m}$, including a towing poll; hence, adequate turning performance is realized owing to the small side surface area and large rudder area. Through simulations, it was verified that the roll convergence was approximately $1^{\circ}$ during turning owing to a relatively large roll damping moment which was due to the large elevator and top surface areas of the hull, as observed in the pure roll tests mentioned in Section 3. The result of a meander test, which evaluates vertical stability in the maneuverability test of the submerged body, is illustrated in Fig. 26. The meander test investigates the stability of motions in the vertical plane direction over time, after the disturbance of a certain size is applied in the vertical plane direction (Jeon et al., 2020). After generating disturbance for a specific time in the vertical plane direction by turning the bow plane and stern elevator, the test examines whether the depth is maintained over time when the elevator is turned again at an angle of $0^{\circ}$. Unlike horizontal plane motions, the vertical plane motions have a restoring moment, which is expressed as the sum of gravitational force and buoyancy, and thus converges to a specific value without diverging to

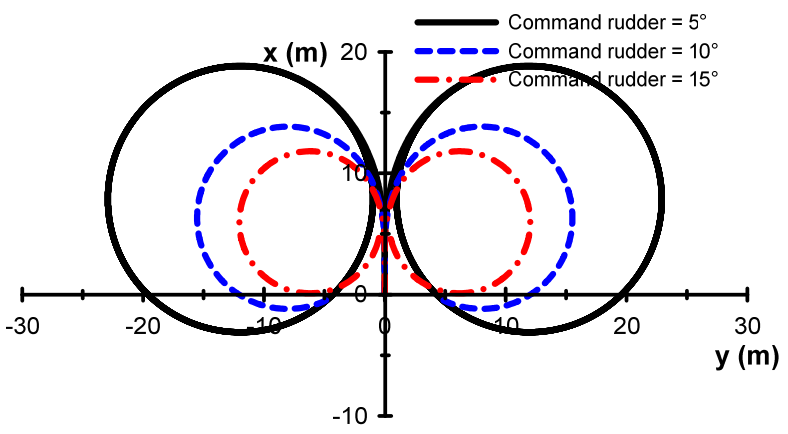

Fig. 24 Trajectories of turning tests
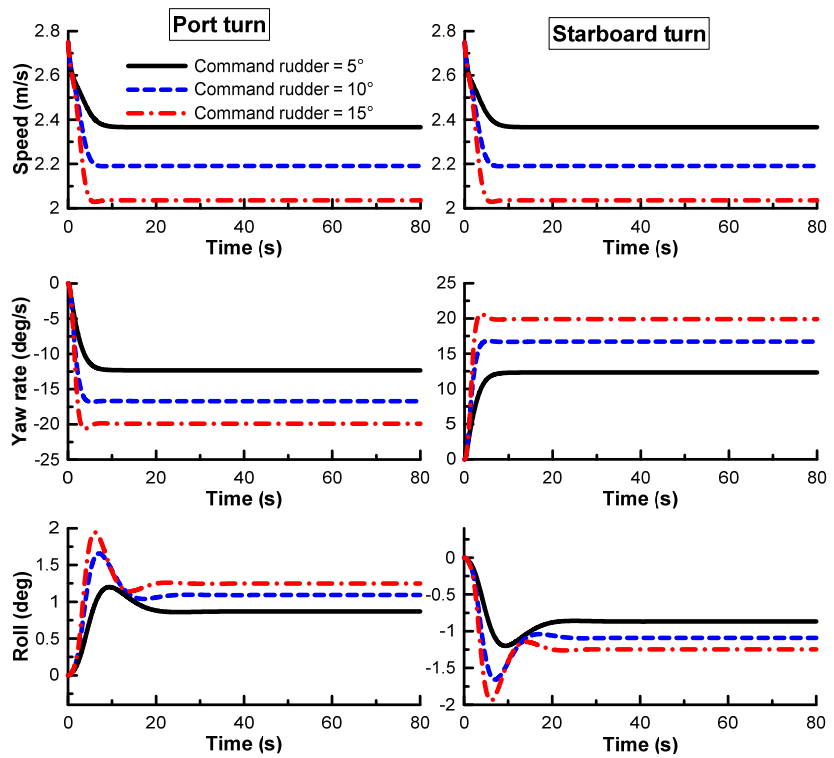

Fig. 25 Motion variables of turning tests

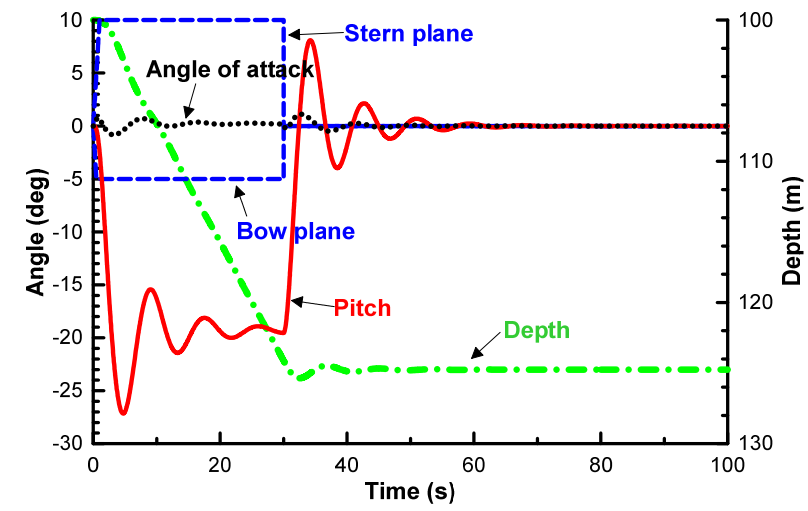

Fig. 26 Meander test

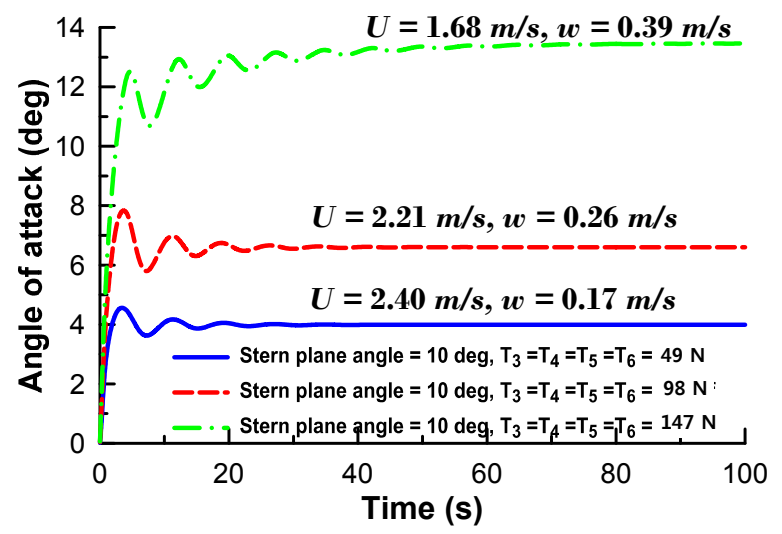

Fig. 27 Large angle of attack test

a pitch angle. When the subject submerged body is assumed to be under neutral buoyancy, the pitch angle converges to zero, and the depth is maintained after the rudder angle is maintained at $0^{\circ}$ after $30 \mathrm{~s}$, as illustrated Fig. 26. Vertical stability can be examined again via a meander simulation, based on the results presented in Fig. 23. The motions in a large angle of attack were simulated by generating thrust and thrust moment in heave and pitch directions, while increasing the 
thrust of auxiliary thrusters $T_{3}, T_{4}, T_{5}$, and $T_{6}$ attached on the hull presented in Fig. 22 to 49 N, 98 N, and 147 N; the obtained results are presented in Fig. 27.

\section{Concluding Remarks}

Maneuvering coefficients for a hydrodynamic model were estimated by performing virtual captive model tests based on CFD for a submerged body that can exhibit large angles of attack and receives various control inputs. The conditions for the virtual captive model tests based on CFD were established by considering that the subject submerged body has a large control panel area and exhibits a large angle of attack motion. A practical inference method was established to verify the results using the linear coefficients of the submerged body because no experimental results were available. Maneuvering coefficients were identified by distinguishing a linear model from a nonlinear model, based on the results of the static test. In general, the determination coefficients of the linear model were closer to 1 than those of nonlinear coefficients in the CFD analysis results, which implied that errors occurred in a mathematical model of hydrodynamic forces as drift angle and angle of attack increased. The accuracy of the mathematical model improved as the order of hydrodynamic derivatives increased; however, the structural stability of the mathematical model of hydrodynamic forces reduced accordingly. The mathematical model was appropriately established because the determination coefficients were generally 0.95 or higher. Because linear coefficients have significant physical implications, the application point of a damping force was verified when a small perturbed motion was generated. Moreover, the application point of the damping force was realistic when the motion of the hull and rotation of the control panel occurred. Dynamic characteristics of the submerged body were evaluated by establishing a mathematical model with six degrees of freedom for thrust generated by six thrusters and hydrodynamic forces applied on the hull by a large angle of attack motion and the rotation of the control panel. Excellent turning characteristics were obtained because the rudder area was larger than the side surface area of the hull, and the stability of the vessel's course was secured. Arbitrary thrust was applied to the auxiliary thrusters attached to the hull, and the large angle of attack motion in the vertical direction was simulated by changing the elevator; however, the effect of the design variables, including the weight of the submerged body and its center of gravity, were greater. The hydrodynamic model will be further improved by tuning various coefficients and comparing the results of an actual experiment with the results of a CFD analysis.

\section{Funding}

This research was financially supported by the Institute of Civil Military Technology Cooperation, funded by the Defense Acquisition Program Administration and Korean government Ministry of Trade, Industry, and Energy.

\section{References}

Bae, J.-Y., \& Sohn, K.-H. (2009). A Study on Manoeuvring Motion Characteristics of Manta-type Unmanned Undersea Vehicle. Journal of the Society of Naval Architects of Korea, 46(2), 114126. https://doi.org/10.3744/snak.2009.46.2.114

Cho, Y.J., Seok, W., Cheon, K.-H., \& Rhee, S.H. (2020). Maneuvering Simulation of an X-plane Submarine Using Computational Fluid Dynamics. International Journal of Naval Architecture and Ocan Engineering., 12, 843-855. https://doi.org/10.1016/j.ijnaoe. 2020.10 .001

Fossen, T. I. (2011). Handbook of Marine Craft Hydrodynamics and Motion Control. New Jersey, USA: John Wiley \& Sons.

Jeon, M., Lee, D., Yoon, H.K., \& Koo, B. (2016). Modeling and Tracking Simulation of ROV for Bottom Inspection of a Ship using Component Drag Model. Journal of Ocean Engineering and Technology, 30(5), 374-380. https://doi.org/10.5574/ksoe. 2016.30.5.374

Jeon, M., Nguyen, T.T., Yoon, H.K., \& Cho, H.J. (2020). A Study on Verification of the Dynamic Modeling for a Submerged Body Based on Numerical Simulation. International Journal of Engineering and Technology Innovation, 10(2), 107-120. https://doi.org/10.46604/ijeti.2020.4350

Jeon, M., Yoon, H.K., Hwang, J., \& Cho, H.J. (2017). Study on Maneuvering Characteristics of Submerged Body by Changing Its Design Parameters. Journal of Ocean Engineering and Technology, 31(2), 155-163. https://doi.org/10.5574/ksoe.2017. 31.2.155

Jung, J.-W., Jeong, J-H., Kim, I-G., \& Lee, S.-K. (2014). Experimental Study on Hydrodynamic Coefficients of Autonomous Underwater Glider Using Vertical Planar Motion Mechanism Test. Journal of Ocean Engineering and Technology, 28(2), 119-125. https:// doi.org/10.5574/ksoe.2014.28.2.119

Kim, Y.-G., Yun, K.-H., Kim, S.-Y., \& Kim, D.-J. (2012). Captive Model Test of Submerged Body Using CPMC. Journal of the Society of Naval Architects of Korea, 49(4), 296-303. https://doi.org/10.3744/snak.2012.49.4.296

Nguyen, T. T., Yoon, H. K., Park, Y., \& Park, C. (2018). Estimation of Hydrodynamic Derivatives of Full-Scale Submarine using RANS Solver. Journal of Ocean Engineering and Technology, 32(5), 386- 392. https://doi.org/10.26748/ksoe.2018.6.32.5.386

Park, J.-Y., Kim, N., Rhee, K.-P., Yoon, H. K., Kim, C., Jung, C., ... Lee, S. (2015). Study on Coning Motion Test for Submerged Body. Journal of Ocean Engineering and Technology, 29(6), 436 -444. https://doi.org/10.5574/ksoe.2015.29.6.436

Quérard, A., Temarel, P., \& Turnock, S. (2008). Influence of Viscous Effects on the Hydrodynamics of Ship-Like Section Undergoing Symmetric and Anti-Symmetric Motions Using RANS. Proceedings of the 27th International Conference on Offshore Mechanics and Arctic Engineering, Estoril, Portugal, 683-692. https://doi.org/10.1115/OMAE2008-57330 
Yeo, D.J., Yoon, H.K., Kim, Y.G., \& Lee, C.M. (2006). Sensitivity Analysis on the Stability of a Submarine Concerning its Design Parameters. Journal of the Society of Naval Architects of Korea, 12, 843-855. https://doi.org/10.1016/j.ijnaoe.2020.10.001

Takashina, J. (1986). Ship Maneuvering Motion due to Tugboats and Its Mathematical Model. Journal of the Society of Naval Architects of Japan, 160, 93-102. https://doi.org/10.2534/ jjasnaoe1968.1986.160_93

Yoon, H.K., \& Kim, S.Y. (2005). A Study on the Model Test Scheme for Establishing the Mathematical Model of Hydrodynamic Force and Moment Acting on a Slowly Moving Ship. Journal of the Society of Naval Architects of Korea, 42(2), 98-104. https://doi.org/10.3744/SNAK.2005.42.2.098

\section{Author ORCIDs}

\section{Author name}

Jeon, Myungjun

Mai, Thi Loan

Yoon, Hyeon Kyu

Ryu, Jaekwan

Lee, Wonhee

$\mathrm{Ku}$, Pyungmo
ORCID

0000-0002-1655-5364

0000-0002-0849-3204

0000-0001-6639-0927

$0000-0003-4176-3867$

0000-0002-1063-3800

0000-0003-1506-9935 